\title{
Selective Innervation of Fast and Slow Muscle Regions during Early Chick Neuromuscular Development
}

\author{
Victor F. Rafuse, Louise D. Milner, and Lynn T. Landmesser \\ Department of Neurosciences, Case Western Reserve University, School of Medicine, Cleveland, Ohio 44106-4975
}

The electrical properties of adult motoneurons are well matched to the contractile properties of the fast or slow muscle fibers that they innervate. How this precise matching occurs developmentally is not known. To investigate whether motoneurons exhibit selectivity in innervating discrete muscle regions, containing either fast or slow muscle fibers during early neuromuscular development, we caused embryonic chick hindlimb muscles to become innervated by segmentally inappropriate motoneurons. We used the in vitro spinal cordhindlimb preparation to identify electrophysiologically the pools of foreign motoneurons innervating the posterior iliotibialis (pITIB), an all-fast muscle, and the iliofibularis (IFIB), a partitioned muscle containing discrete fast and slow regions. The results showed that the pITIB and the fast region of the IFIB were exclusively innervated by motoneurons that normally supply fast muscles. In contrast, the slow region of the IFIB was always innervated by motoneuron pools that normally supply slow muscles. Some experimental IFIB muscles lacked a fast region and were innervated solely by "slow" motoneurons. In addition, the intramuscular nerve branching patterns were always appropriate to the fast-slow nature of the muscle (region) innervated. The selective innervation was found early in the motoneuron death period, and we found no evidence that motoneurons grew into appropriate muscle regions, but failed to form functional contacts. Together, these results support the hypothesis that different classes of motoneurons exhibit molecular differences that allow them to project selectively to, and innervate, muscle fibers of the appropriate type during early neuromuscular development.

Key words: motoneuron; muscle; neuromuscular development; axon guidance; specificity; selective innervation; spinal cord preparation
For the nervous system to function properly, developing neurons must extend axons over long distances and synapse with their correct targets. In the peripheral nervous system, it is well recognized that motoneuron pools selectively innervate specific muscles from the onset with relatively few errors (Landmesser, 1992). It is less clear whether subsets of developing motoneurons within a pool selectively recognize and synapse with different classes of muscle fibers (Grinnell, 1995). Adult motoneurons are generally classified into different classes the electrical properties of which are well matched to the contractile properties of the fast or slow muscle fibers that they innervate (Kernell, 1992). Studies involving cross-reinnervation in mature animals have shown that many of the molecular and contractile properties of muscle fibers can be determined by the innervating motoneuron through its imposed pattern of activation (Vrbova et al., 1995). Nevertheless, such observations do not address directly how matching is achieved during initial development. Does it occur by a similar mechanism, or are there predetermined classes of fast and slow motoneurons and muscle fibers that are matched by some form of recognition process?

\footnotetext{
Received May 14, 1996; revised Aug. 1, 1996; accepted Aug. 6, 1996.

This study was supported by National Institutes of Health Grant NS19640 from the National Institute of Neurological Diseases and Stroke. V.R. was supported by the Rick Hansen Man in Motion Legacy Foundation, and L.M. was supported by National Institutes of Health Training Grant T32 4D07104 from the National Institute of Child Health and Human Development. We thank Roman Fatkhiyev for his technical assistance and Drs. Shilpi Banerjee and Juan Bruses for critical reading of this manuscript.

Correspondence should be addressed to Dr. Lynn Landmesser, Department of Neurosciences, Case Western Reserve University, 10900 Euclid Avenue, Cleveland, OH 44106-4975.

Copyright (C) 1996 Society for Neuroscience $0270-6474 / 96 / 166864-14 \$ 05.00 / 0$
}

During early muscle development, myofiber type differentiation is autonomous to the limb, does not require neural input (Butler et al., 1982; Phillips and Bennett, 1984; Condon et al., 1990b; Fredette and Landmesser, 1991), and is not altered by subsequent motoneuron innervation (Condon et al., 1990a; Fredette and Landmesser, 1991). These observations are compatible with the hypothesis that developing motoneurons selectively synapse with the appropriate class of muscle fiber type. Support for this hypothesis comes from developmental studies showing that early postnatal rodent motor units are strongly biased toward a given fiber type (Thompson et al., 1984; Gordon and Van Essen, 1985; Fladby and Jansen, 1988). However, technical limitations make it difficult to extend such studies in mammals to earlier developmental stages when motor axons make initial contacts with developing muscle fibers. Thus, it remains undetermined whether motoneurons selectively innervate specific fiber types from the onset, or whether death of mismatched motoneurons and/or synaptic rearrangements contribute to the observed fiber type bias.

The avian neuromuscular system has several advantages for such studies. First, fast and slow primary myotubes are segregated into distinct fast and slow regions that are distributed in a characteristic spatial pattern (McLennan, 1983; Crow and Stockdale, 1986; Vogel and Landmesser, 1987). Second, early in ovo experimental manipulations can be performed to alter the position of developing muscles to test whether motoneurons make selective guidance choices on the basis of muscle fiber type. Finally, chick fast and slow muscle regions have characteristic patterns of intramuscular nerve branching that determine the distribution of neuromuscular synapses (Dahm and Landmesser, 1988).

To investigate whether motoneurons exhibit some selectivity in 
innervating fast and slow muscle regions and whether the pattern of intramuscular branching is imposed by the innervating nerve, we caused embryonic chick muscles to become innervated by segmentally inappropriate motoneurons. We focused on the innervation of two muscles: the posterior iliotibialis (pITIB), which contains only fast fibers, and the iliofibularis (IFIB), which has distinct fast and slow fiber regions. We found that foreign motoneuron pools exhibited considerable selectivity when innervating these two muscles; the slow region of the IFIB was innervated by pools that normally project to muscles predominantly composed of slow primary myotubes, whereas the fast regions of the IFIB and the pITIB were selectively innervated by pools that normally project to muscles containing predominantly fast primary myotubes.

Preliminary results have been published previously in abstract form (Rafuse et al., 1995).

\section{MATERIALS AND METHODS}

Embryonic surgery. All embryos were incubated at $39^{\circ} \mathrm{C}$ in a circulated air incubator. Experimental manipulations were performed on St. 17-18 (Hamburger and Hamilton, 1951) White Leghorn chick embryos. Anteriorposterior (A-P) hindlimb rotations were performed as described previously in detail (Vogel and Landmesser, 1987). Briefly, a window was made in the egg with a dental drill to expose the embryo. The vitelline membrane and amnion were cut, and the embryo was stained with a sterile solution of $1 \%$ neutral red in distilled water. One of the developing hindlimb buds was carefully removed with flame-sharpened tungsten needles (0.077 mm wire; World Precision Instruments, Sarasota, FL), reversed along its A-P axis, and fastened onto the contralateral side of a host embryo after removal of the contralateral limb bud. This caused the A-P axis to be reversed while maintaining normal orientation of the dorsal-ventral axis. Operated embryos were moistened with sterile Tyrode's solution, and the window in the egg was sealed with a sterile coverslip and paraffin. Operated embryos were placed back in the incubator and were allowed to develop for another 5-7 d before the final acute experiment.

In some cases, transplanted limb buds either failed to adhere or developed into malformed limbs. However, in many cases limbs were completely normal in form but were rotated about the A-P axis. For the most part, only limbs in which the posterior dorsal part (that region containing the IFIB and pITIB) was normally patterned were utilized for the experiments reported here. In addition, we have included some embryos that had a specific but interesting alteration in pattern. Although the pITIB and slow region of the IFIB were normally formed, the fast (posterior) region of the IFIB was completely lacking. This alteration was seen at early stages and may have occurred because the myogenic precursor cells that normally populate this region were lacking from the transplanted limb bud (C. Lance-Jones, personal communication). This type of defect was associated in fact with a failure to transplant the most posterior lateral region of the donor limb bud. As described in Results, such "all-slow" IFIBs were used to distinguish among the mechanisms responsible for generating the selective innervation found.

Electromyogram (EMG) and muscle nerve recordings. In vitro spinal cord-limb preparations and EMG recordings from St. 32-36 chick embryos were performed as described in detail by Landmesser and O’Donovan (1984a). Briefly, embryos were quickly decapitated, eviscerated, and placed in cooled, well oxygenated Tyrode's solution. A ventral laminectomy was performed to expose the spinal cord. Skin and connective tissue surrounding the spinal nerves and hindlimb muscles were carefully removed for subsequent stimulation and EMG recordings. The spinal cord-limb preparation was maintained for several hours in well oxygenated Tyrode's solution $\left(30^{\circ} \mathrm{C}\right)$, after which the motoneurons became spontaneously active. The EMG activation patterns from experimental muscles in the rotated limb were recorded simultaneously with EMGs from control muscles in the contralateral limb to identify electrophysiologically the group of motoneurons innervating the different regions of the IFIB and pITIB muscles.

Previous studies have shown that each motoneuron pool in the embryonic lumbosacral (LS) spinal cord has a highly stereotyped pattern of activation that is characterized both by the duration of the burst and by the length of the inhibitory period at the onset of each cycle (Landmesser and O'Donovan, 1984a; O'Donovan, 1989). It has also been shown that such stereotyped activation patterns are not altered when a motoneuron pool is caused to innervate a foreign muscle after early A-P limb bud rotations as in the present study (see also Landmesser and O'Donovan, 1984b). In the isolated cord preparation, homologous muscles in both limbs are normally activated in synchrony. Thus, it is possible to deduce the motoneuron pool(s) contributing to a given experimental muscle, both by its internal bursting characteristics and by its phase relationship to the contralateral control muscles.

Previous studies have shown that the electrophysiological identification of motoneuron pools using the criteria described above is accurate and correlates well with anatomical characterization of pools based on their position within the spinal cord after retrograde labeling from the muscle (Landmesser and O'Donovan, 1984b; Vogel and Landmesser, 1987; Vogel, 1988). Thus, it is possible to detect when a muscle is innervated by foreign motoneurons that have bursting characteristics that differ from the muscles' normal activation patterns. Similarly, from the EMG recordings, it is also possible to detect when a muscle is innervated by mixtures of motoneurons with differing activation patterns (Vogel, 1988). It should be pointed out that chick myotubes do not conduct action potentials at the developmental stages used in this study and that the recorded EMG responses are in fact postsynaptic potentials (Landmesser and O'Donovan, 1984a). Because this "patch-type" recording (see below) with tight-sealing suction electrodes can even detect spontaneous miniature postsynaptic potentials (Landmesser and O'Donovan, 1984a), this method avoids the complication of failing to detect subthreshold responses. We cannot exclude the possibility of failing to detect a small number of motoneurons projecting to a given region, but our recordings should have detected any significant contribution.

Because individual motoneuron pools are located in highly characteristic segmental levels, when recording from muscles we often directly stimulated individually the LS nerves to determine the segmental origin of the motoneurons innervating the regions from which the EMGs were recorded. The relative contribution each LS spinal nerve made to the innervation of each region was determined by stimulating each spinal nerve with a suprathreshold maximal stimulus and by recording the amplitude of the compound postsynaptic response evoked with the same suction electrode that was used to record the EMG. In other cases, postsynaptic potentials were not recorded, but the source of the innervation was estimated by visual observation of twitch contractions. In the tables displaying these data, the spinal nerve that contributed the majority of the innervation, based on electrophysiological recordings, is shown in bold and is underlined. In some cases, the anatomical arrangement of the spinal nerves and adjacent vertebrae precluded the stimulation of each nerve separately. An ampersand ("\&") was used to indicate when stimulation was of a common trunk containing two spinal nerves.

A sequence of hindlimb movements was initiated every 8-12 min, either spontaneously or by a single electrical stimulus applied to the thoracic region of the spinal cord with a polyethylene suction electrode. Fine-tip suction electrodes were made by pulling polyethylene tubing (PE-190; Clay Adams, Parsippany, NJ) over a small flame. Typical recording electrodes have an inner diameter of 50-100 $\mu \mathrm{m}$. Single-pulse electrical stimuli were administered with a standard stimulator (Grass S88, Quincy, MA) that was isolated from ground with a stimulator isolation unit (Grass PSIU6B). EMG patterns were recorded using similar suction electrodes, amplified with a bandwidth between $3 \mathrm{~Hz}$ and 10 $\mathrm{kHz}$, displayed on an oscilloscope (Tektronix R5030, Beaverton, OR) and chart recorder (Gould, Cleveland, $\mathrm{OH}$ ), and stored on analog tape (Vetter, Rebersburg, PA) for later analysis. Tight electrode seals made with these flexible tapered suction electrodes allow for stable recordings from contracting muscles for long periods of time (for additional details of recording procedures, see Landmesser and O'Donovan, 1984a). In some embryos, the neural activity was recorded from the cut proximal end of the experimental IFIB nerve after it had been pulled into a tight-fitting suction electrode. Recorded muscle nerve signals were amplified and stored in a manner similar to the EMG activation patterns.

Electrophysiological data analysis. To identify the innervating motoneuron pools, the EMG and muscle nerve activation patterns were quantified as described previously (Landmesser and O'Donovan, 1984a). Briefly, activity was measured in cycles, one cycle being defined as the period between the onset of synchronous discharge activity. The preceding and following burst cycles were divided into $40 \mathrm{msec}$ intervals, and the number of times the muscle was active during each interval was used to construct a frequency histogram. Muscle activity preceding the onset of each bursting cycle is only included in the histogram if the muscle is active 
during this time period. Thus, the frequency histogram describes the probability that a muscle will be active at any given time before, and after, the onset of each bursting cycle.

Immunostaining. Immediately after cessation of the EMG recording session, the experimental and contralateral control muscles were quickly removed and prepared for either muscle whole mounts or frozen-section immunostaining.

Muscle whole mounts. Muscles chosen for whole mounts were washed in PBS, fixed for $2 \mathrm{~min}$ in ice-cold acetone, washed several times in PBS (5-10 $\mathrm{min} /$ wash), incubated in primary antibody (full-strength hybridoma supernatant) with $0.3 \%$ Triton-X for $1.5 \mathrm{hr}$ (room temperature), washed in PBS, fixed for $20 \mathrm{~min}$ in $3.7 \%$ formaldehyde-PBS, washed in PBS, incubated overnight $\left(4^{\circ} \mathrm{C}\right)$ with an appropriate fluorescein-conjugated secondary antibody, washed in PBS and, finally, mounted between two glass coverslips in $50 \%$ glycerin-PBS containing $0.03 \mathrm{mg} / \mathrm{ml}$ $p$-phenylenediamine (Sigma, St. Louis, MO) to prevent fading.

The IgG monoclonal antibody $(\mathrm{mAb}) \mathrm{C}-2$, which recognizes a neurofilament epitope, was used to visualize the pattern of intramuscular branching (Dahm and Landmesser, 1988). A goat anti-mouse fluoresceinconjugated secondary antibody (Sigma) was used to visualize the C-2labeled axons.

Frozen-section immunohistochemistry. Muscles chosen for frozen sections were mounted in O.C.T. (Miles, Elkhart, IN) and quickly frozen in isopentane that was cooled with dry ice. Fourteen micrometer sections were taken from the proximal region of the hindlimb containing the IFIB and pITIB muscles and dried on previously subbed glass slides. Sections were serially incubated with primary antibodies that exclusively recognize either fast or slow muscle fibers. Sections were incubated for $1.5 \mathrm{hr}$ (room temperature) with 5D2 (a monoclonal $\mathrm{IgG}$ antibody that recognizes fast muscle fiber sarcoplasmic reticulum $\mathrm{Ca}^{2+}$ ATPase; Kaprielian and Fambrough, 1987) (kindly provided by D. Fambrough, The Johns Hopkins University, Baltimore, MD), washed in PBS, incubated for $1.5 \mathrm{hr}$ (room temperature) with goat anti-mouse rhodamine-conjugated secondary antibody (IgG; Boehringer Mannheim, Indianapolis, IN), washed in PBS, incubated for $1.5 \mathrm{hr}$ (room temperature) with $\mathrm{S} 58$ (a monoclonal $\mathrm{IgA}$ antibody that recognizes chick slow myosin heavy chain; Crow and Stockdale, 1986) (kindly supplied by F. E. Stockdale, Stanford University, Stanford, CA), washed in PBS, incubated for $20 \mathrm{~min}$ in $3.7 \%$ formaldehyde-PBS, washed in PBS, incubated overnight $\left(4^{\circ} \mathrm{C}\right)$ with goat anti-mouse fluorescein-conjugated secondary antibody (IgA; Cappell, West Chester, PA), washed in PBS and, finally, mounted in 50\% glycerinPBS containing $0.03 \mathrm{mg} / \mathrm{ml} p$-phenylenediamine.

Alternating sections were stained with a $\mathrm{mAb}, 5 \mathrm{E}$, that recognizes all isoforms of the neural cell adhesion molecule (NCAM) (Frelinger and Rutishauser, 1986) (provided by U. Rutishauser, Case Western Reserve University, Cleveland, $\mathrm{OH}$ ) to visualize the entire patterning of muscles and nerves in the experimental and contralateral control limbs.

\section{RESULTS}

\section{Surgically produced foreign innervation of the pITIB and IFIB muscles}

The pITIB, an "all-fast" muscle, and the IFIB, with distinct fast and slow regions, are located in the posterior lateral portion of the thigh and are innervated from the sciatic plexus (Fig. 1A). The pITIB motoneuron pool is located in LS segments 4 and 5, whereas that of the IFIB is located in LS4-LS7. Both pools are located at the lateral edge of the lateral motor column (Fig. $1 \mathrm{~A}$, Spinal cord $X$-section). After an A-P limb rotation, these muscles become located anteriorly in the limb and are innervated by motoneurons, the pools of which originate in LS1-LS3 and ordinarily form the crural plexus (see also Landmesser and O'Donovan, 1984b). When these "anterior" axons grow into the posterior regions of the limb, they form an anatomical nerve branching pattern appropriate for the sciatic plexus (Landmesser and O'Donovan, 1984b).

Previous studies have shown that after A-P limb rotations (Lance-Jones and Landmesser, 1980, 1981b; Landmesser and O'Donovan, 1984b) motoneurons that normally innervate muscles derived from the dorsal muscle mass will preferentially innervate foreign muscles that are also derived from the dorsal muscle mass.
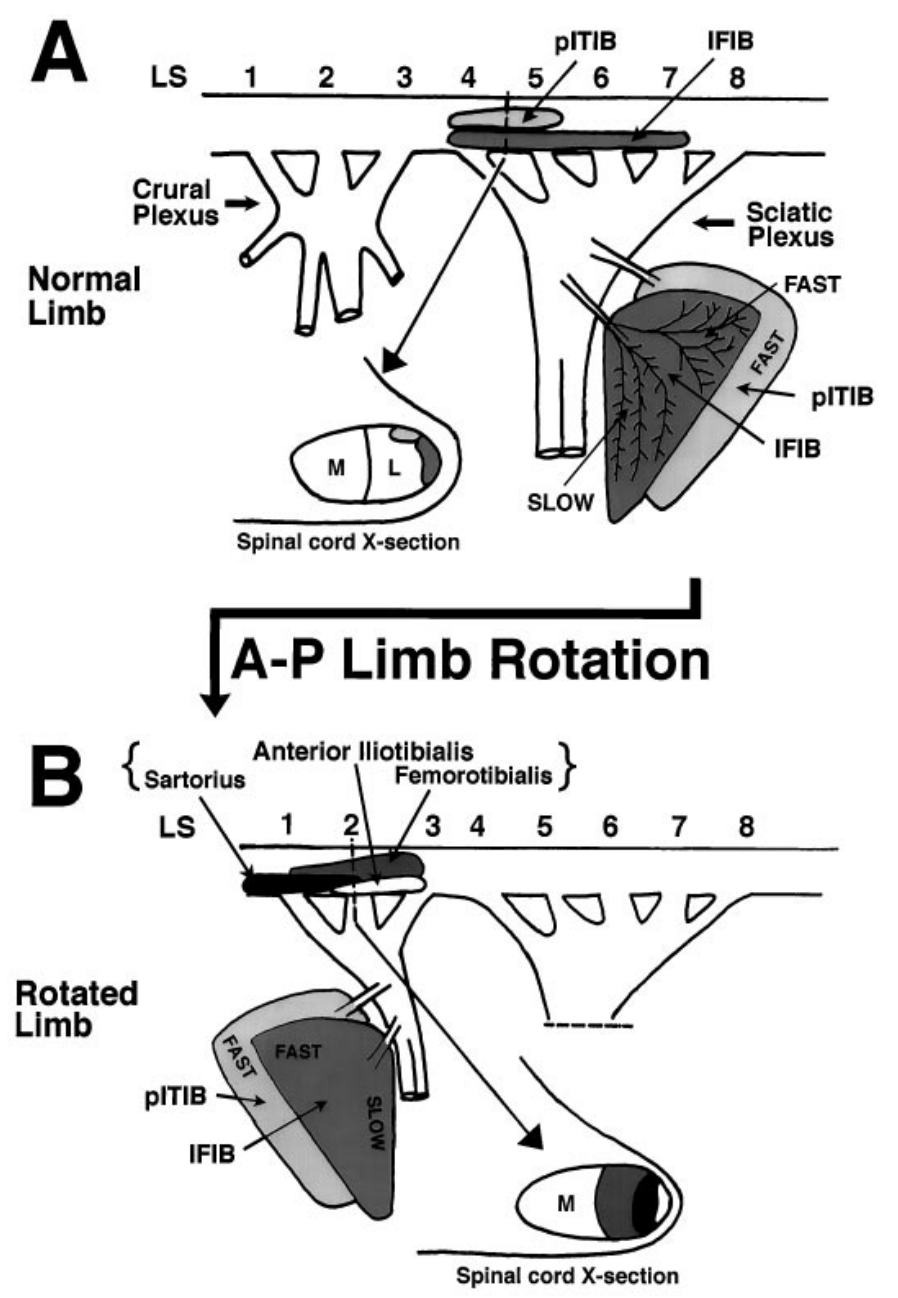

Figure 1. Schematic representation of the pITIB and IFIB innervation in normal $(A)$ and experimental $(B)$ embryos after A-P limb bud rotation. $A$, In normal embryos, both muscles are innervated by motor axons located in the posterior sciatic nerve. The $P I T I B$ motoneuron pool is located in $L S 4-L S 5$, and the IFIB motoneuron pool extends throughout $L S 4-L S 7$. Both motoneuron pools are located in the lateral edge of the lateral motor column (Spinal cord X-section). B, After A-P limb bud reversal, both muscles are displaced rostrally and are innervated by axons whose motoneuron pools normally form the crural plexus (i.e., $L S 1-L S 3$ ). The three motoneuron pools most likely to innervate the $p I T I B$ and $I F I B$ muscles in the rotated limbs are the sartorius, anterior iliotibialis, and femorotibialis, all of which are located laterally within the lateral motor column (Spinal cord $X$-section). IFIB, Iliofibularis; $p I T I B$, posterior iliotibialis; $A-P$, anterior-posterior; $M$, medial; $L$, lateral.

Thus, the three major "dorsal" motoneuron pools in LS1-LS3 that would be expected to potentially innervate the rotated IFIB and pITIB muscles are the sartorius (SART), the femorotibialis (FT), which consists of several separate heads, and the anterior iliotibialis (aITIB) (Fig. $1 B$ ).

Figure $2 A$ shows a cross section through the midthigh of a St. 35 embryo stained with an NCAM-specific antibody to visualize the muscles innervated by LS1-LS3 (left of dashed line) and all other muscles and nerves that compose the developing chick thigh. The pITIB and IFIB can also be distinguished as two distinct muscles located dorsal to the sciatic nerve (Fig. 2A; black stars indicate sciatic nerve). An adjacent section, stained with a slow myosinspecific antibody (Fig. 2B), shows that the SART and anterior portion of the aITIB are predominantly composed of slow fibers 


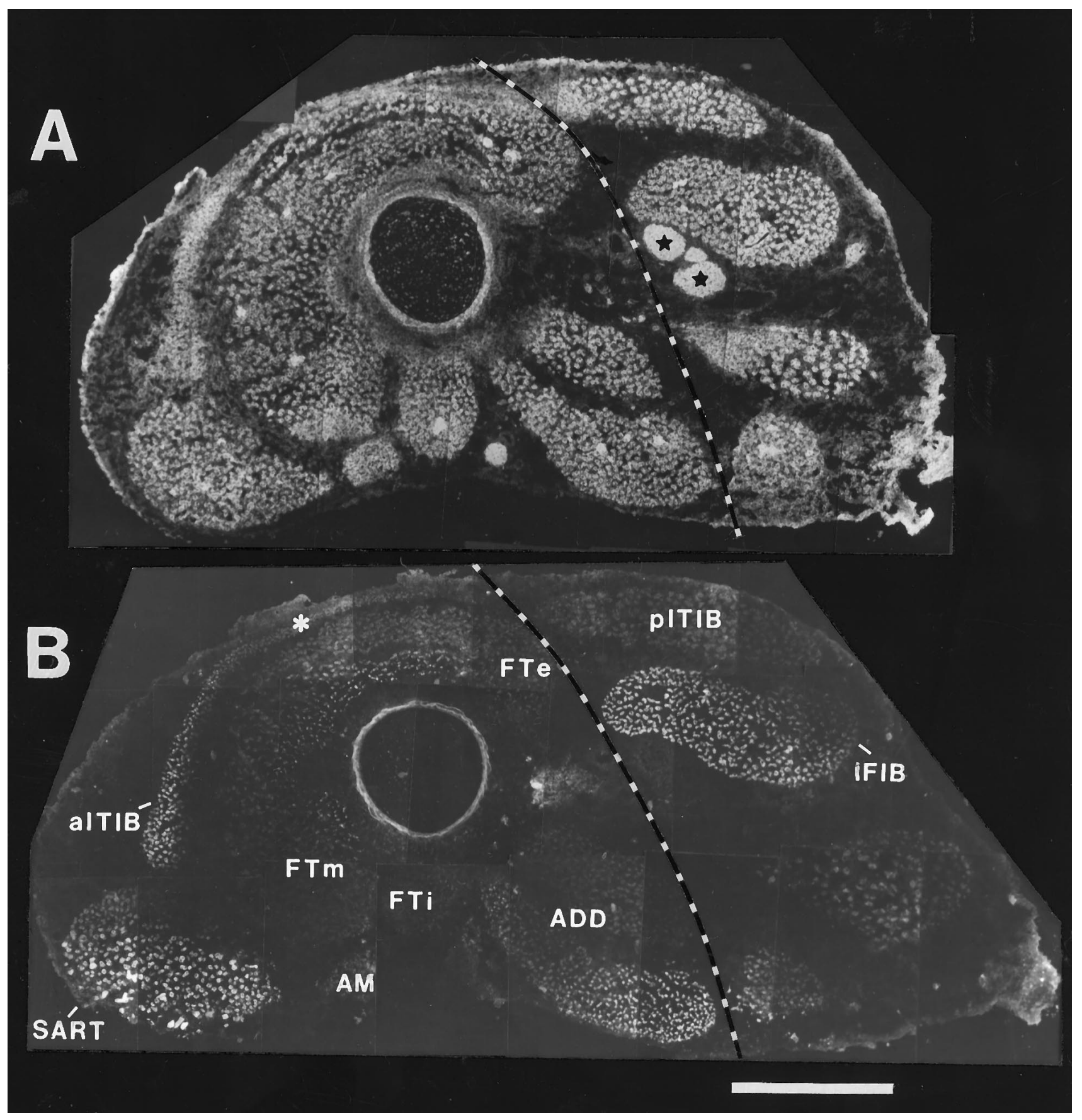

Figure 2. Immunostaining for NCAM (with 5E) and slow muscle myosin (with S58) shows the developing muscles in the normal chick thigh that contain slow myotubes. In both panels, the dorsal side is up and anterior is left. A, At St. 35, all myotubes and intramuscular and sciatic nerve trunks (stars) are NCAM-immunopositive. B, S58 immunostaining shows that the sartorius ( $A A R T)$, anterior portion of the anterior iliotibialis (aITIB), iliofibularis $(I F I B)$, and one head of the adductor $(A D D)$ contain slow myotubes. Of the muscles innervated from LS1-LS3 (those to the left of the dashed line), the $S A R T$ and one head of the $A D D$ are predominantly composed of slow fibers, the aITIB has both a slow and a fast (asterisk) muscle fiber region, whereas femorotibialis muscles $(F T)$ contain predominantly fast fibers. Note that motoneurons innervating the ADD muscles are not expected to innervate the dorsally derived IFIB and posterior iliotibialis ( $p I T I B)$ muscles in A-P rotated limbs. $A D D$, Adductor; $A M$, ambiens; $F T e$, femorotibialis externus; $F T i$, femorotibialis internus; FTm, femorotibialis medius; $S A R T$, sartorius; $a I T I B$, anterior iliotibialis; $p I T I B$, posterior iliotibialis; IFIB, iliofibularis. Scale bar, $500 \mu \mathrm{m}$.

and thus, by definition, are innervated by pools containing "slow" motoneurons. The adductor pool, which innervates a fast as well as a slow head (Fig. $2 B ; A D D$ ), would not be expected to innervate the dorsally derived IFIB and pITIB in the rotated limb because these neurons selectively project to ventrally derived muscles (Landmesser and O'Donovan, 1984b). The muscles not stained by the slow myosin $\mathrm{mAb}$ in Figure $2 B$ are predominantly fast and can be distinguished by staining with a mAb selective for fast $\mathrm{Ca}^{2+}$-ATPase (Kaprielian and Fambrough, 1987; Fredette and Landmesser, 1991) (for example, see Fig. 6B). These "fast" motoneuron pools include the three heads of the FT (the femorotibialis externus, FTe; femorotibialis internus, FTi; and femorotibialis medius, FTm; as well as 

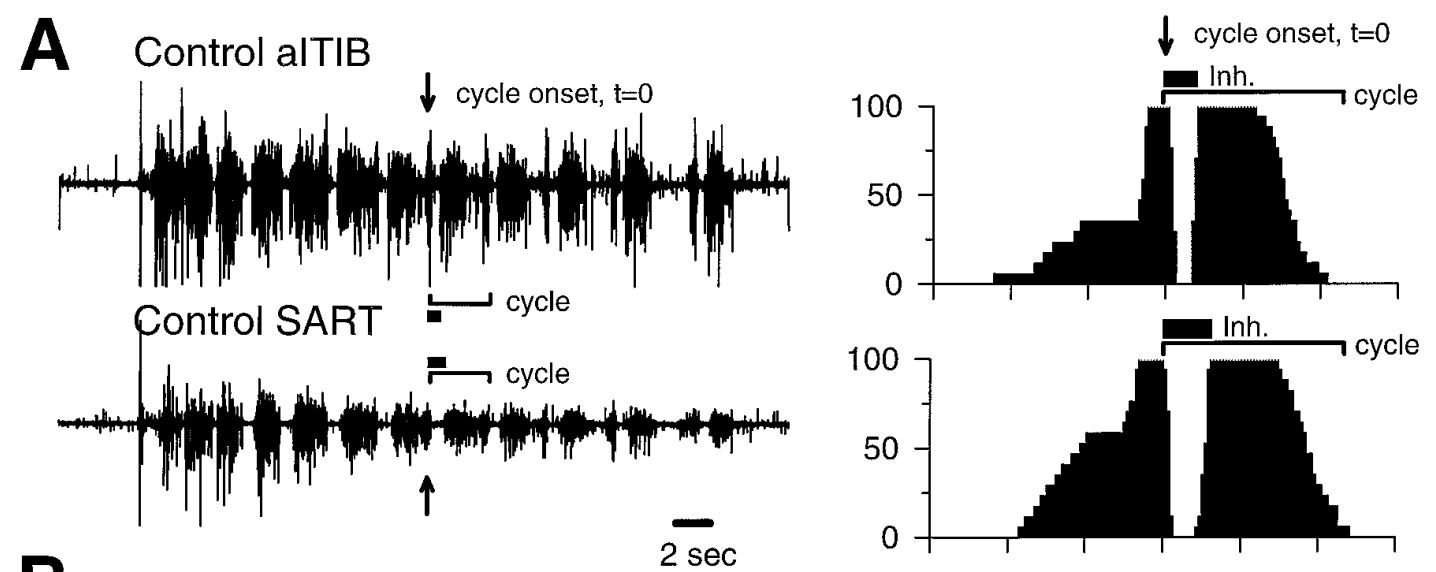

B

\section{Control FTm}

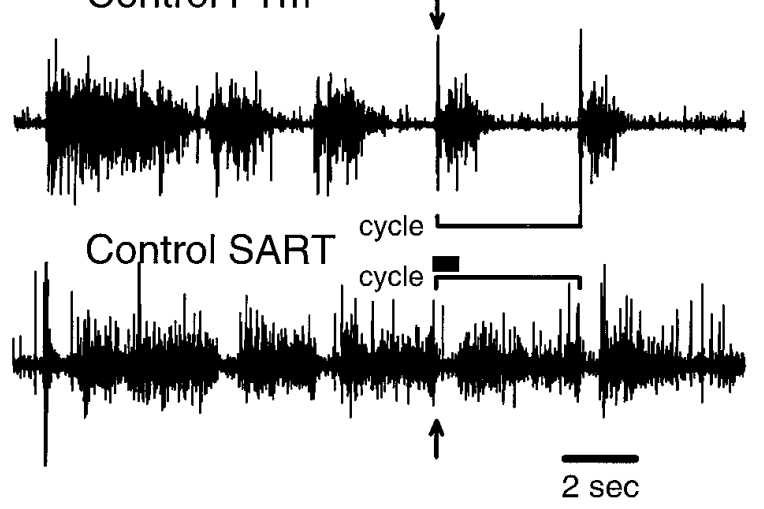

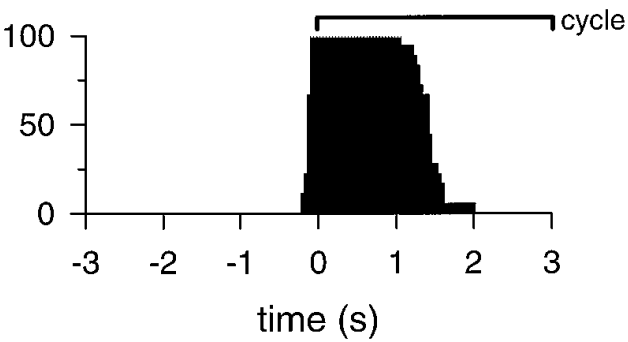

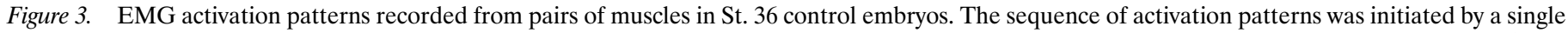

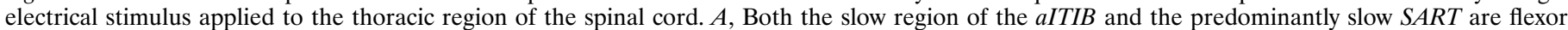

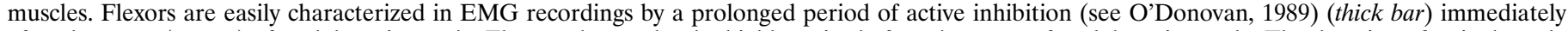

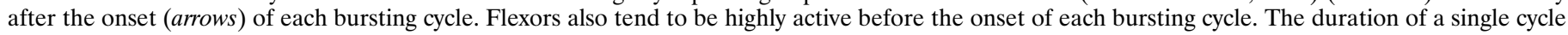

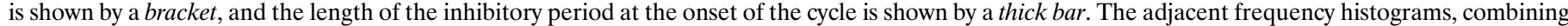

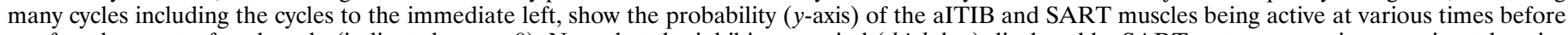

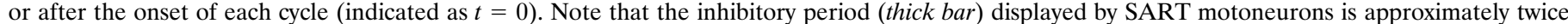

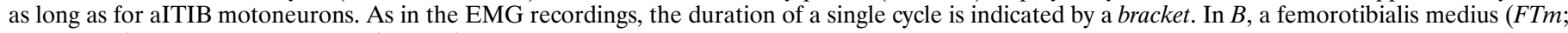

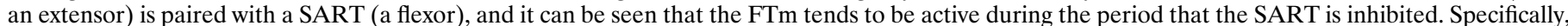

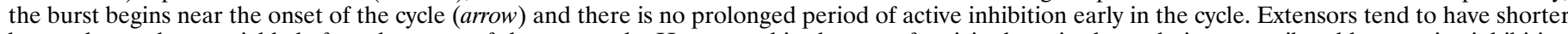

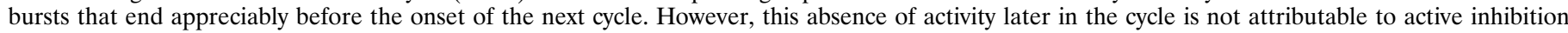

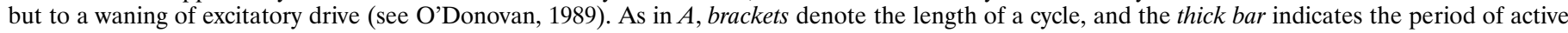

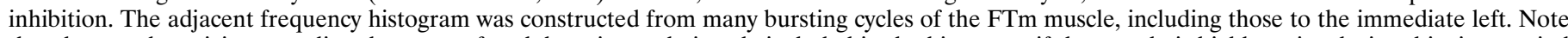

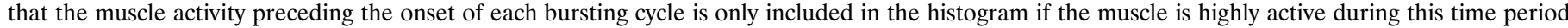
(e.g., SART muscle highly active, whereas the FTm is inactive). inh, Inhibitory period (see Fig. 2 for additional abbreviations).

the ambiens, AM) (see also McLennan, 1983; Vogel and Landmesser, 1987). In addition, the aITIB has a very thin posterior region that is all fast (Fig. 2B, asterisk).

\section{Electrophysiological identification of motoneurons}

To test whether these "fast" and "slow" motoneuron pools would selectively innervate the fast and slow regions of the pITIB and IFIB muscles in the A-P rotated limbs, we determined the source of innervation contributing to each muscle region using the in vitro isolated spinal cord-limb preparation. In the isolated spinal cord preparation, each motoneuron pool has a characteristic and highly stereotyped activation pattern (Landmesser and O'Donovan, 1984a,b). In addition, the stereotypic activation patterns were not altered even when the developing axons were forced to innervate foreign muscles after early A-P limb rotations (Landmesser and O'Donovan, 1984a,b; Vogel, 1987), as was done in the present study. These results indicate that the activation patterns of developing motoneurons are not altered by retrograde myotypic influ- ences from the muscle but, rather, are intrinsically determined within the spinal cord (Landmesser and O'Donovan, 1984a,b) before axon outgrowth as part of the developmental program that determines a motoneuron's identity (Lance-Jones and Landmesser, 1980). We therefore made use of these patterns to identify electrophysiologically the motoneuron pool(s) innervating the experimental IFIB and pITIB muscles. After a single stimulus to the thoracic cord, the central pattern generator in the LS region of the cord gives rise to a series of "step" cycles in which both hindlimbs move in synchrony. Typical EMG activation patterns for three of the pools that could contribute innervation to the experimental IFIB and pITIB are shown in Figure 3. The SART and anterior portion of the aITIB are both composed of slow muscle fibers and, therefore, by definition are innervated by slow motoneurons. The SART and anterior portion of the aITIB are activated as flexors as distinguished by the prolonged inhibitory period immediately after the onset of each cycle (Fig. $3 A$, thick 

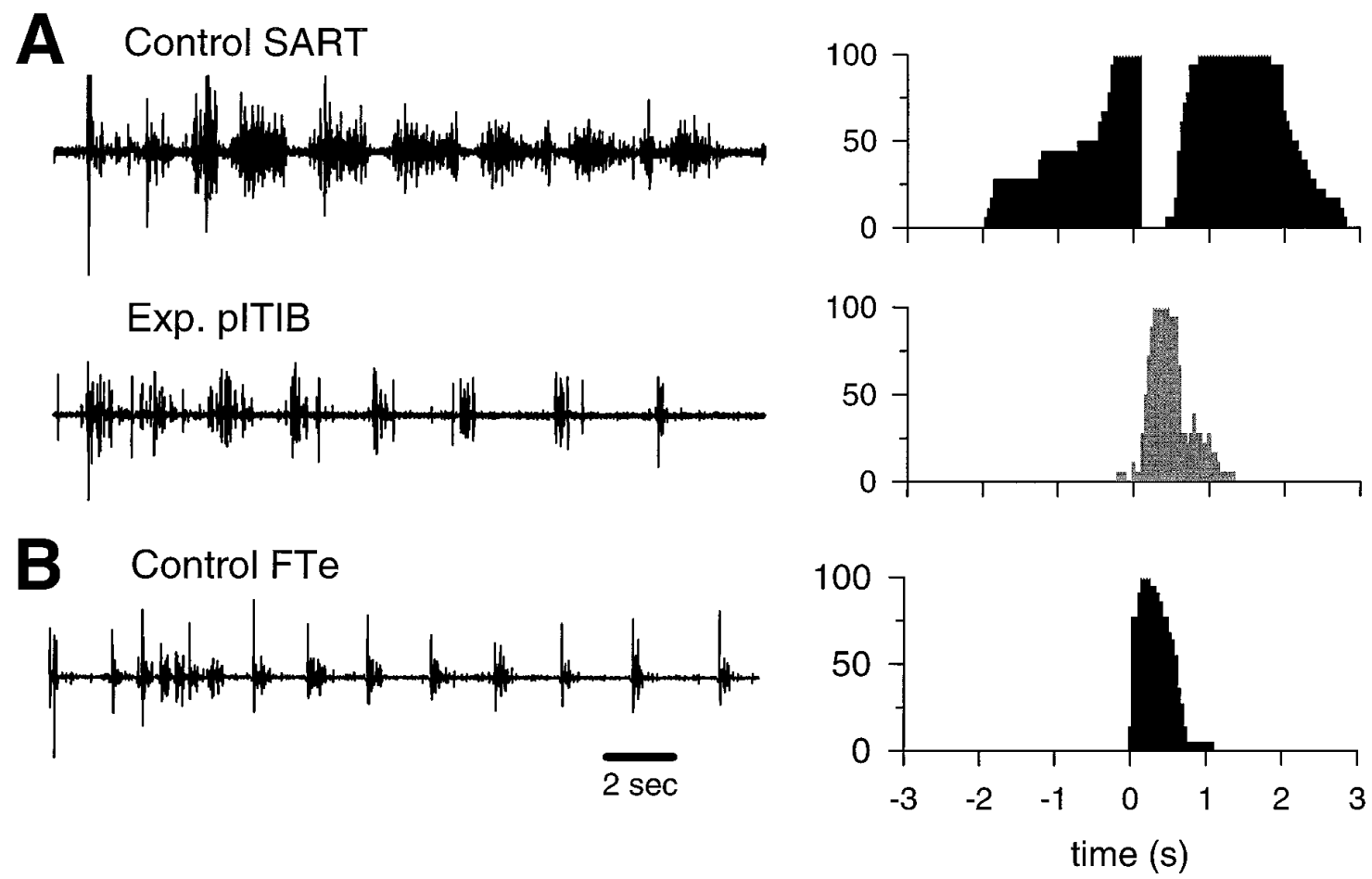

Figure 4. Typical EMG activation patterns and corresponding probability of activation histograms (controls: filled; experimental: shaded), recorded from an experimental pITIB (Exp. pITIB), paired with the contralateral Control SART muscle $(A)$, and compared with an FTe activation pattern (Control FTe) recorded from a control embryo of a similar stage $(B)$ (see Fig. 2 for abbreviations).

bar). After the period of inhibition, both muscles are generally active until the onset of the next bursting cycle [see Materials and Methods and Landmesser and O'Donovan (1984a) for additional details of the functional characterization of motoneuron pools]. However, the SART and aITIB EMG activation patterns can be distinguished from one another by the significantly longer period of inhibition displayed by SART motoneurons. This is seen most clearly when the bursts are displayed as histograms reflecting the probability of activity at any point in the burst cycle (Fig. $3 A$, adjacent histograms; see Materials and Methods for details). The mean inhibitory period for SART motoneurons is approximately twice as long as that for anterior aITIB motoneurons.

In contrast, extensor muscles, such as the FTm (Fig. $3 B$ ), are characterized by the absence of activity before the onset of each bursting cycle and a very short period of inhibition (not obvious on this time scale) that is immediately followed by a burst of characteristic duration depending on the muscle. Although the activation patterns of the different extensor muscles vary somewhat with respect to their burst duration and length of the inhibitory period (for example, see Figs. 4, 5B,C); all of them can clearly be distinguished from the two flexor muscles studied, the SART and aITIB. As shown in Figure 3B, the flexor and extensor muscles are alternately active throughout a sequence of evoked muscle activity (i.e., compare FTm and SART EMG in Fig. 3B). By combining the criteria of when a motoneuron pool is active in the step cycle, the burst duration, and the length of the inhibitory period, it is possible to distinguish between the motoneuron pools that can potentially contribute to the innervation of the experimental IFIB and pITIB. Most important, the activation patterns of all pools innervating fast muscles, which could potentially innervate our experimental muscles (i.e., AM, FTe, FTi, FTm, and posterior portion of the aITIB), are easily distinguished from the activation patterns of the two pools innervating slow muscles (i.e., anterior portion of the aITIB and SART).

\section{Electrophysiological identification of foreign motoneurons innervating muscles in rotated hindlimbs}

Figure $4 A$ shows a typical rhythmic EMG bursting pattern recorded from an experimental pITIB muscle, in a rotated hindlimb, paired with the contralateral control SART muscle. In this embryo, the experimental pITIB was clearly activated by extensor motoneurons when compared with the control SART activation pattern. Specifically, the pITIB did not exhibit an obvious inhibitory period and was activated completely out of phase with the control SART (compare histograms in Fig. 4A). This pattern of activity was very similar to the FTe muscle activation pattern recorded from a control embryo of a similar stage (Fig. 4B). Ten out of fourteen experimental pITIB muscles displayed this pattern of activity, three had activation patterns similar to control FTm muscles, and one was similar to either the FTi or the AM (FTi/ AM) muscle (Table 1). Thus, all of the experimental pITIB muscles recorded from were innervated by motoneurons normally innervating fast muscles. None displayed flexor-like activity and, therefore, none was innervated by "slow motoneurons" that normally supply the aITIB or SART muscles.

Motoneuron pools innervating FT muscles in normal embryos are located in LS1-LS3, the majority of motoneurons being distributed in LS2 and LS3 (Landmesser, 1978). Consistent with the FT activation patterns, all experimental pITIB muscles received innervation from motoneuron pools located in LS3, whereas a few were innervated from motoneurons in LS1 and LS2 (Table 1). Visual localization of twitch contractions in two embryos indicated that the experimental pITIB was innervated by motoneurons from LS3 and LS4 and in one embryo from LS3-LS5 (Table 
A
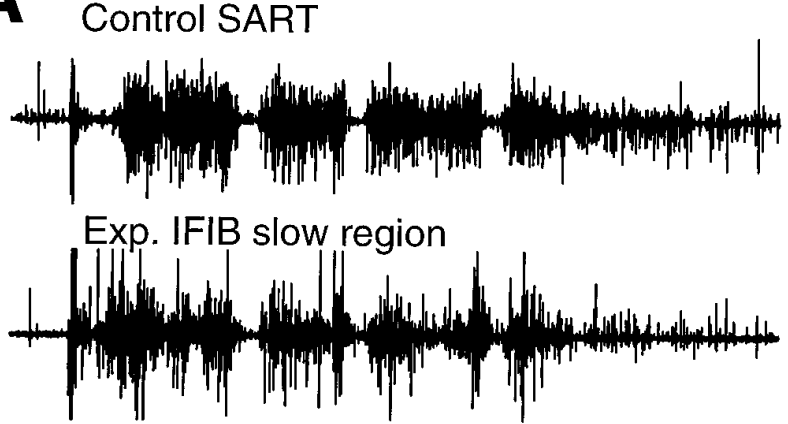

B

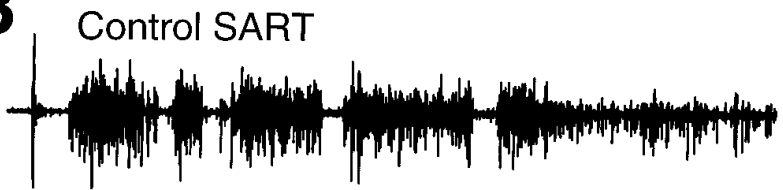

Exp. IFIB fast region

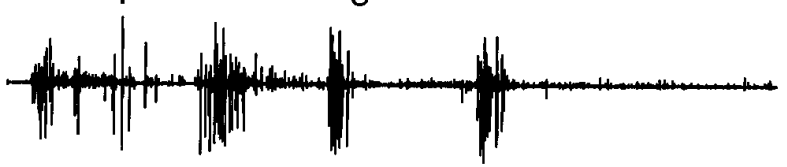

C Exp. IFIB fast region

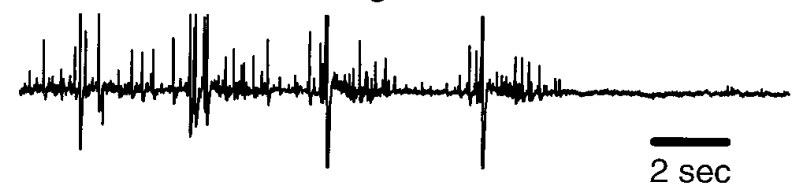

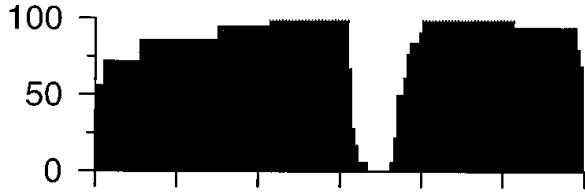
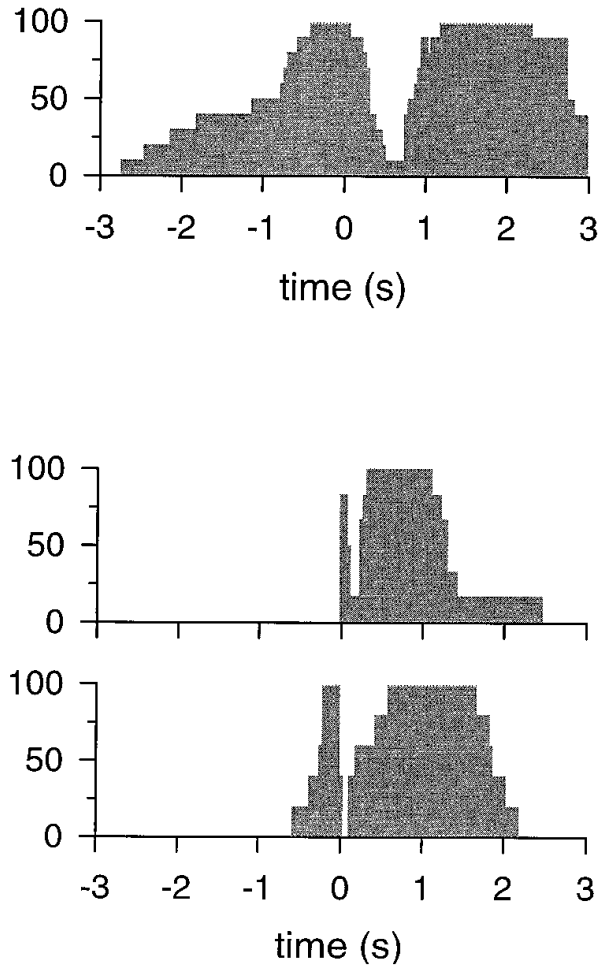

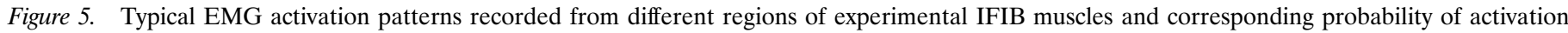

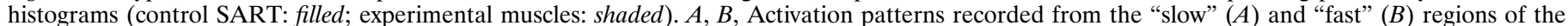

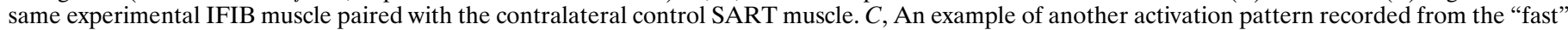

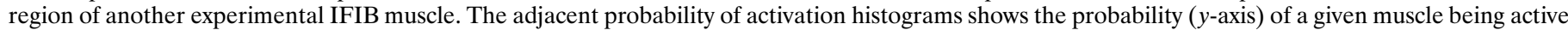
at various times before or after the onset of each bursting cycle (see Fig. 2 for abbreviations).

1). In all three cases, LS4 (and LS5 for RL1 4-16) entered the sciatic plexus and, thus, these experimental pITIB muscles may also have been innervated by their own pITIB motoneurons (normally located in LS4 and LS5; see Fig. 1 $A$ ).

The experimental IFIBs were especially interesting in that the fast and slow regions were selectively innervated by different pools of motoneurons. A typical experimental IFIB recording is shown in Figure 5, $A$ and $B$, and is paired with the contralateral control SART muscle. In this embryo, the "slow" region of the IFIB was activated in a flexor-like manner, very similar to the activation pattern of the contralateral control SART muscle (Fig. 5A). In striking contrast, the "fast" region of the same muscle was clearly activated in an extensor-like activation pattern similar to that of FT motoneurons (Fig. 5B). These results are shown more quantitatively in the probability of activation histograms in Figure 5. In all five cases in which we were able to record successfully from both the fast and the slow regions of the IFIB, the "slow" region was activated in a SART-like pattern whereas the "fast" region was never innervated by SART and/or aITIB motoneurons (Table 2). Although the pools innervating the "fast" region varied (FTe, or a pattern consistent with either the FTi or the AM, FTi/AM; Table 2), they were all activation patterns characteristic of fast muscles. Figure $5 C$ shows an example of an FTi/AM activation pattern, recorded from the fast region of another experimental IFIB, which can easily be distinguished from the SART or aITIB patterns by the short inhibitory period and length of burst after the onset of each bursting cycle (see histogram in Fig. $5 C$ ).

The EMG activation patterns indicated that the different muscle regions were innervated by discrete pools of motoneurons. SART motoneurons are normally located in LS1 and LS2, whereas FT motoneurons are predominantly located in LS2-LS3 (Landmesser, 1978). Consistent with the EMG activation patterns, the slow region of the experimental IFIB muscles was predominantly innervated by motoneuron pools located in LS1 and LS2, whereas the fast region tended to be innervated by motoneurons from LS2 and LS3 (Table 2), which agrees with the FT activation patterns recorded from that region. However, three embryos received some innervation ( $<12 \%$ of the total) from LS3 (LR1 10-17, LR1 4-16, and LR2 9-12; Table 2) and one from LS4 (LR1 4-16). These motoneurons, therefore, could not be from the SART pool. Similarly, the fast region in one embryo (LR1 4-16) received innervation from LS4 which is segmentally inappropriate for FT motoneurons. It is possible that, in these embryos, the slow region was also innervated by IFIB motoneu- 


\begin{tabular}{|c|c|c|c|}
\hline Embryo & Innervation & Activation pattern & St. \\
\hline LR1 12-5 & $3^{*}$ & FTe & 33 \\
\hline LR3 10-4a & $\underline{\mathbf{3}}$ & FTm & 34 \\
\hline LR3 9-6 & NR & FTe & 35.5 \\
\hline LR3 11-30 & $3 \& 4^{*}$ & FTm & 35.5 \\
\hline LR3 9-18 & $1 \& 2, \underline{\mathbf{3}}$ & FTe & 36 \\
\hline LR3 12-5 & $1 \& 2, \underline{\mathbf{3}}$ & FTe & 36 \\
\hline RL1 3-13 & $1, \underline{\mathbf{2}}, 3$ & FTi/AM & 36 \\
\hline LR1 10-17 & $\mathrm{NR}$ & FTe & 36 \\
\hline LR1 1-3 & $3,4^{*}$ & FTe & 36 \\
\hline LR1 10-19 & $3^{*}$ & FTe & 36 \\
\hline RL1 3-22 & $3^{*}$ & FTe & 36 \\
\hline LR1 4-16 & $3,4 \& 5^{*}$ & FTm & 36 \\
\hline RL2 12-13 & $2,3^{*}$ & FTe & 36.5 \\
\hline LR2 9-12 & $3^{*}$ & FTe & 36.5 \\
\hline
\end{tabular}

Underlined, bold numbers indicate the LS nerve that contained the predominant number of motoneurons innervating the muscle as deduced from electrical recordings. *, Source of innervation estimated only by visual localization of twitch contractions and not with direct electrical recording. "\&" indicates simultaneous stimulation of both LS nerves when it was not possible to stimulate each separately (see Materials and Methods for additional details). NR, Not recorded; FTe, femorotibialis externus; FTi, femorotibialis internus; FTm, femorotibialis medius; AM, ambiens.

rons that projected to their correct muscle target while the fast region was innervated by other motoneurons with bursting characteristics similar to FT motoneurons. The bursting characteristic of IFIB motoneurons would not have allowed them to be distinguished within the SART EMG activation patterns (IFIB muscles are normally only active for a very brief period at the onset of each bursting cycle; Landmesser and O'Donovan, 1984a). Alternatively, the experimental IFIB muscles may have been innervated by LS3 and LS4 motoneurons whose bursting activity was below the level of detection using the EMG recordings. However, as noted in Materials and Methods, the type of EMG recording used in this study is very sensitive and should have detected any motoneurons that made a significant contribution to the muscle innervation.

In summary, these results clearly show that both the experimental pITIB and the fast region of the IFIB were innervated by motoneuron pools that normally project to fast muscles. They were never innervated by the "slow" SART or aITIB pools. In contrast, the EMG recorded from the slow region of the IFIB had an inhibitory period and activation pattern that were very similar in pattern and duration to those of the EMG recorded from the contralateral SART muscle. If any of the "fast" pools had innervated this region to any significant extent (see Materials and Methods), the motoneurons would have fired during the prolonged SART inhibitory period and, therefore, would have been easily detected.

Motoneurons innervating several mammalian muscles are topographically ordered such that motoneurons at more rostral levels of the spinal cord systematically innervate muscle fibers located at more anterior levels (Swett et al., 1970; Brown and Booth, 1983; Laskowski and Sanes, 1987). At present, the mechanisms involved in this type of matching are unknown. One possibility is that motoneurons simply innervate muscle regions based on the spatial location of their axons within the nerve trunk. This type of specific innervation would not necessitate specific targeting signals to guide axons to the correct muscle regions. More rostral motoneurons would simply innervate anterior mus- cle regions, whereas more caudal motoneurons would innervate more posterior regions. However, this simple spatial topography cannot explain the selective innervation observed in the present study. Normally, motoneurons innervating the pITIB are located in LS4 and LS5, whereas those supplying the IFIB are topographically located in LS4-LS7; the most posterior ("fast") region receives innervation from the most caudal motoneurons within the IFIB pool. After performing an A-P limb bud rotation, we found that the predominant motoneuron innervation to the experimental pITIB and fast region of the IFIB was from LS2 and LS3, whereas the slow region of the IFIB was innervated predominantly by motoneurons from LS1 and LS2 (Tables 1, 2). If the innervation to the rotated limbs was simply spatially determined, one would expect that the pITIB and fast region of the IFIB (now anatomically in a rostral position) would be innervated by the most rostral LS motoneurons (i.e., LS1 and LS2; the spatially closest segments) whereas the slow IFIB region (now anatomically caudal) would be innervated by more caudal motoneurons (i.e., LS3; see Fig. $1 B$ for orientation). None of the embryos displayed this type of segmental innervation, indicating that cellular mechanisms other than spatial topography were most likely responsible for the selective innervation observed in this study.

\section{Immunocytochemical identification of muscle fiber types in rotated hindlimbs}

To confirm that the regions of the PITIB and IFIB from which EMGs were recorded actually contained the expected complement of either fast or slow muscle fibers, the muscles in some of the rotated limbs were removed immediately after cessation of the electrophysiological recordings and prepared for immunocytochemical staining. Alternate serial cross sections of the limb were either immunostained for NCAM (to visualize both muscle and nerves and to aid in anatomically identifying the muscles in the rotated limbs) or double-labeled with S58 and 5D2, mAbs that selectively label chick slow and fast fibers, respectively (see Materials and Methods). Figure 6 shows the distribution of slow (Fig. $6 A$ ) and fast (Fig. 6B) myotubes from one experimental hindlimb where completely different activation patterns were recorded from the anterior and posterior regions of the IFIB (embryo LR1 4-16; Table 2). The "SART-like" activation pattern was recorded from the anterior region of the IFIB muscle (Fig. $6 \mathrm{~A}$, open arrow), the "FTe-like" EMG was recorded from a more posterior site from the same muscle (Fig. 6A, filled arrow), and an "FTm-like" was recorded from the overlying pITIB. As expected, the "SARTlike" activation pattern was recorded from a region of the IFIB that was confirmed to be predominantly composed of slow primary myofibers. On the other hand, the "FT-like" EMGs were recorded from fast muscle fibers located in both the pITIB and the posterior region of the IFIB.

\section{Intramuscular nerve branching patterns in experimental muscles}

Previous studies have shown that fast and slow avian muscles/ muscle regions have distinct patterns of intramuscular nerve branching that determines the distribution of neuromuscular synapses (Dahm and Landmesser, 1988; Landmesser et al., 1988). The main intramuscular nerve branches supplying slow muscles, including the slow region of the IFIB, grow longitudinal to the muscle fibers all the way to the distal end of the muscle without branching. Nerve ramification in the slow region occurs as small collateral sprouts that extend at regular intervals along the main nerve trunks (Fig. $7 A$, arrowhead). This pattern of intra- 
Table 2. Innervation and activation pattern of "partitioned" IFIB muscles in rotated limbs

\begin{tabular}{|c|c|c|c|c|c|}
\hline \multirow[b]{2}{*}{ Embryo } & \multicolumn{2}{|l|}{ "Slow region" } & \multicolumn{2}{|l|}{ "Fast region" } & \multirow[b]{2}{*}{ St. } \\
\hline & Activation pattern & Innervation & Activation pattern & Innervation & \\
\hline RL2 9-10 & SART & $1 \& 2$ & FTe & $\underline{\mathbf{3}}$ & 34.5 \\
\hline LR1 10-17 & SART & $1 \& 2,3$ & FTi/AM & $1 \& 2,3$ & 36 \\
\hline LR1 4-16 & SART & $\overline{1, \underline{2}, 3}, 4$ & FTe & $\overline{2, \underline{\mathbf{3}}, 4}$ & 36 \\
\hline LR2 9-12 & SART & $1, \underline{2}, 3$ & FTi/AM & NR & 36.5 \\
\hline
\end{tabular}

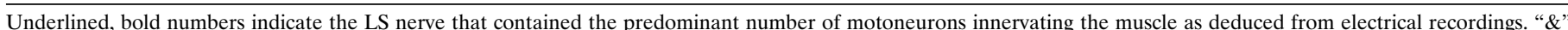

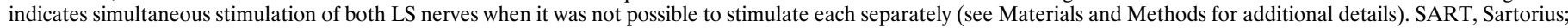
FTe, femorotibialis externus; FTi, femorotibialis internus; AM, ambiens; NR, not recorded.

muscular nerve branching allows each motor axon to multiply innervate a single slow muscle fiber. In contrast, axons supplying the fast region of the IFIB muscle (Fig. $7 A$, bracket) grow transversely to the myotubes (Fig. $7 A$, arrow) and undergo a characteristic reductive branching pattern where each subsequent branch is smaller in diameter than the previous one. This pattern of nerve branching favors focal innervation of each fast muscle fiber by a single motor axon.

To determine whether this characteristic pattern of intramuscular nerve branching is established in muscles innervated by foreign motoneurons, the muscles in some of the rotated limbs were removed immediately after cessation of the electrophysiological recordings and prepared for whole-mount neurofilament

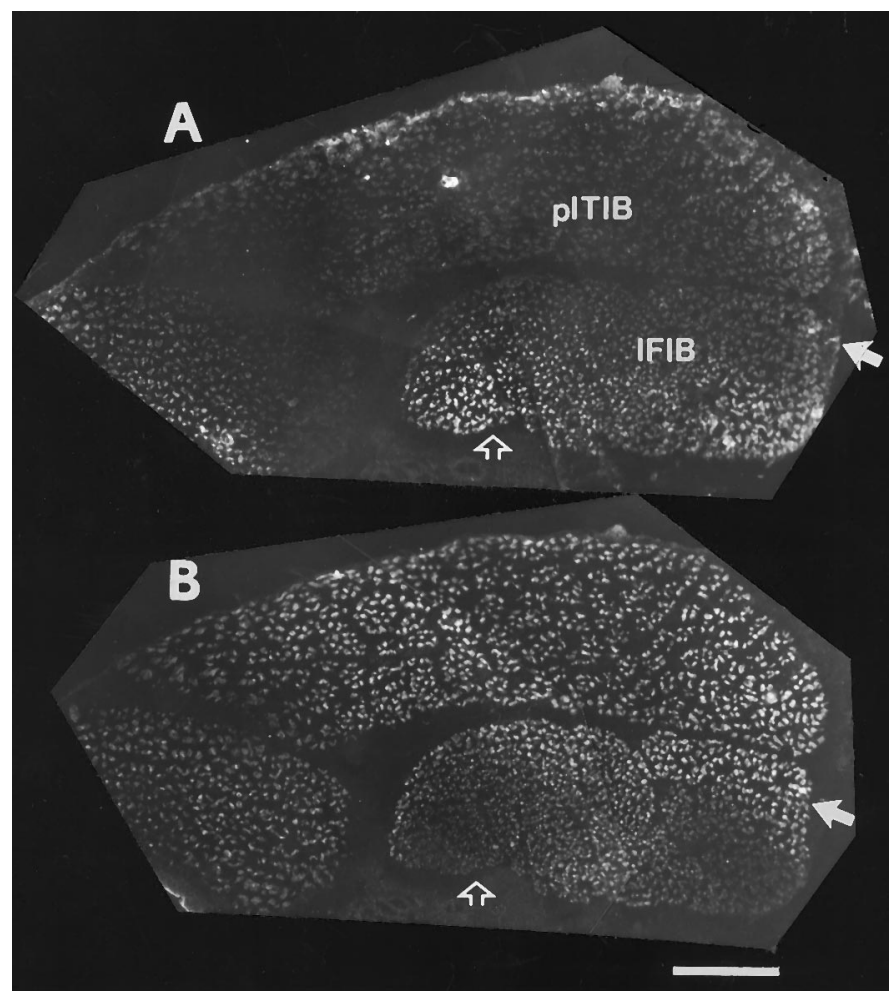

Figure 6. Double immunostaining showing the distribution of slow and fast myotubes in experimental pITIB and IFIB muscles in a rotated hindlimb. Slow muscle myosin $(A)$ and fast muscle $\mathrm{Ca}^{2+}$ ATPase $(B)$ double immunostaining indicates that the "SART"-like activation pattern was recorded from a region of the muscle containing predominantly slow myotubes (open arrow). In contrast, the "FTe"-like pattern was recorded from fast muscle fibers located in the posterior region of the IFIB (filled arrow). pITIB, Posterior iliotibialis; IFIB, iliofibularis. Scale bar, $250 \mu \mathrm{m}$. immunostaining. As shown by the whole mount in Figure $7 B$, the intramuscular branching pattern in the experimental IFIB was similar to normal despite the fact that it was formed by foreign motoneurons arising from spinal nerves LS1-LS3 (Fig. 7B). As in control IFIB muscles, the main nerve trunks supplying the slow region grew parallel to the myotubes and extended small collateral sprouts at regular intervals to multiply innervate the slow muscle fibers (Fig. 7B, arrowhead). The main nerve trunk (Fig. 7B, arrow) supplying the fast region (indicated by bracket) grew transverse to the myotubes and underwent characteristic reductive branching.

As shown in the whole mount in Figure $7 C$, the major intramuscular nerve trunks innervating a normal, all-fast, pITIB muscle are restricted to the central region of the muscle and grow obliquely across the myotubes before ramifying and undergoing reductive branching (Fig. $7 C$, arrow perpendicular to muscle fibers). As discussed above, this pattern of intramuscular branching is consistent with the adult pattern of focally distributed synapses in fast avian muscles. The main intramuscular nerves innervating experimental pITIB muscles in rotated hindlimbs were also restricted to the central region of the muscle and grew obliquely across the myotubes (Fig. $7 D$, arrow perpendicular to muscle fibers). However, the few experimental pITIB muscles examined for nerve branching tended to be supplied by many fewer axons than in controls. This might explain why the entire width of the muscle was not innervated.

\section{EMG activation patterns recorded from experimental IFIBs lacking the "fast" muscle region}

In some embryos, the experimental IFIB appeared to have a normal slow region but lacked the fast posterior region that normally gives this muscle its triangular shape (Table 3). As a result, such muscles were thin and strap-like. This absence of the fast region appeared to be caused by failure to excise the most posterior dorsal region of the donor limb at the time of the limb ablation (see Materials and Methods). In some cases, it was observed at early developmental stages (e.g., LR1 9-10; Table 3) and, therefore, did not result from degeneration of muscle fibers that were not innervated. We believe that such muscles may have been produced by interfering with the subpopulation of myogenic precursor cells that would normally populate this region (C. Lance-Jones, personal communication). The intramuscular nerve branching patterns of these muscles, shown in the example in Figure $8 A$, had the typical slow pattern of longitudinally oriented main nerve trunks with collateral side branches, but lacked any reductive branching innervating the posterior region. In accord with this observation, these muscles were also composed almost entirely of slow muscle fibers (Fig. $8 B, C$ ). 


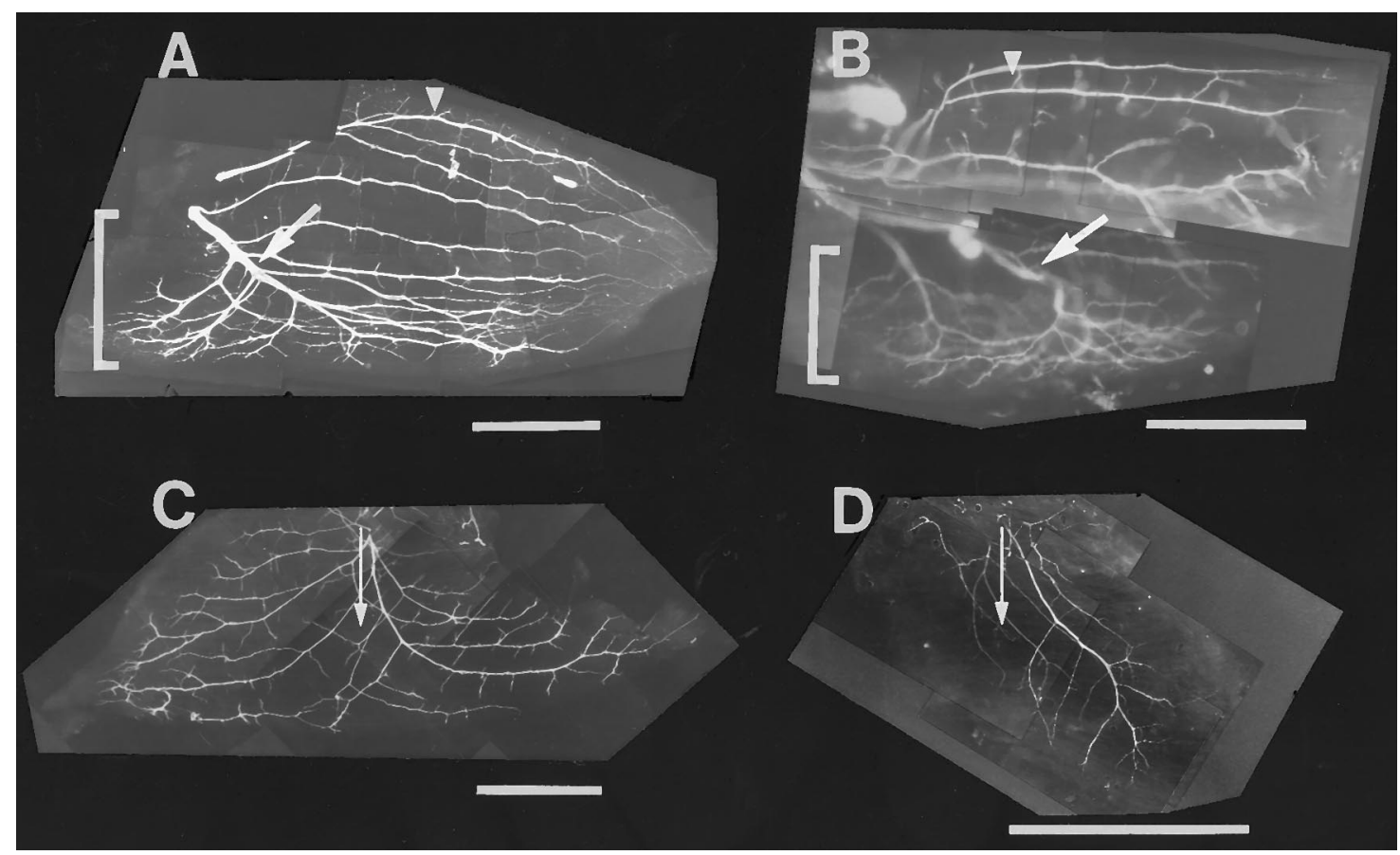

Figure 7. The distribution of intramuscular nerves in the IFIB and pITIB muscles innervated by normal or foreign motoneurons. St. 36 whole-mount preparations of the mixed fast/slow IFIB $(A, B)$ and all-fast pITIB $(C, D)$ in normal $(A, C)$ and A-P rotated hindlimbs $(B, D)$. In all four panels, the muscle fibers run from left (proximal) to right (distal). $A$, In the slow region of the IFIB, the intramuscular nerve trunks grow parallel to the myotubes and intermittently extend short collateral sprouts (arrowhead), whereas the nerve trunk projecting to the fast region (indicated by bracket) is transversely oriented to the fibers and undergoes reductive branching (arrow). B. In IFIB muscles, in A-P rotated hindlimbs, the intramuscular nerve trunks also grow longitudinal to the myotubes in the slow region and intermittently extended small collateral sprouts (arrowhead). In contrast, the main trunk (arrow) projecting to the fast region (indicated by bracket) is oriented transverse to the fibers and undergoes reductive branching. Note the extensive ramification of axons transverse to the muscle fibers in the fast region of the IFIB. $C$, Typical of fast muscles, the main intramuscular nerve trunks in the fast pITIB muscle grow perpendicular (indicated by arrow) to the myotubes and give off a series of reductive nerve branches. $D$, In pITIB muscles in the A-P rotated hindlimbs, the major nerve trunks also grow perpendicular to the myotubes (arrow) and undergo reductive branching. However, unlike normal pITIB muscles, all regions of the experimental pITIB were not innervated, possibly because the muscle was supplied by fewer motoneurons. Scale bars, 1 mm.

A typical EMG activation pattern recorded from an IFIB lacking a fast region is shown in Figure $9 A$, where it is paired with the contralateral control SART muscle. Regardless of where the EMG recording was made, the activation pattern was the same and was indistinguishable from that of the control SART. Similar results were obtained from eight other muscles (see Table 3) and clearly indicate that these thin IFIB muscles were exclusively innervated by SART motoneurons. As expected from the SARTlike EMG activation patterns, the thin IFIB muscles were predominantly innervated by motoneurons from LS1 and LS2. In only one case (LR3 3-22; Table 3) did we observe another type of activation pattern. In this embryo, the muscle appeared to be innervated by motoneurons that normally innervate the slow region of the aITIB.

These unusual IFIB muscles provided us with a means of distinguishing between several distinct mechanisms that could have produced the "selective" innervation we found with EMG recordings. One possibility is that distinct motoneuron pools were selectively guided to innervate the fast and slow muscle regions. Alternatively, matching may have occurred simply because fast and slow motoneurons were spatially located within the sciatic nerve in such a way that the most likely positional targets for innervation were the fast and slow regions of the experimental IFIB. However, this simple explanation seems highly unlikely. If spatial positioning was the only factor determining the fast-slow innervation, one would expect that the "all-slow" IFIB muscles either would be innervated by a mixture of fast and slow motoneu- rons or would be randomly innervated by discrete pools of fast or slow motoneurons. Even though a majority of motor axons innervating dorsal muscles of the chick thigh innervate fast muscles, all of the "all-slow" IFIB muscles were innervated by pools of motoneurons that normally innervate slow muscles (Table 3). These results strongly suggest that slow motoneurons pools selectively grew to and innervated the "all-slow" IFIB muscles.

Table 3. Innervation and activation patterns of "all-slow" IFIB muscles in rotated limbs

\begin{tabular}{llll} 
Embryo & Activation pattern & Innervation & St. \\
\hline LR1 $9-10$ & SART & $1,2^{*}$ & 32 \\
LR1 $12-5$ & SART & $1,2^{*}$ & 33 \\
LR4 $11-30$ & SART & $\underline{\mathbf{1}}, 2$ & 34.5 \\
LR1 $9-12$ & SART & $\underline{\mathbf{1}}$ & 35 \\
LR3 3-22 & aITIB (slow region) & $1,2^{*}$ & 35 \\
LR3 9-6 & SART & $1,2^{*}$ & 35.5 \\
LR3 11-30 & SART & $1,2^{*}$ & 35.5 \\
LR3 9-18 & SART & $\underline{\mathbf{1}} \mathbf{2}, 3$ & 36 \\
LR3 $12-5$ & SART & $\underline{\mathbf{1}}, 2$ & 36 \\
RL2 $12-13$ & SART & $1^{*}$ & 36.5
\end{tabular}

Underlined, bold numbers indicate the LS nerve that contained the predominant number of motoneurons innervating the muscle as deduced from electrical recordings. *, Innervation estimated by visual localization of twitch contractions. "\&" indicates simultaneous stimulation of both LS nerves when it was not possible to stimulate each separately (see Materials and Methods for additional details). SART, Sartorius; aITIB, anterior iliotibialis. 


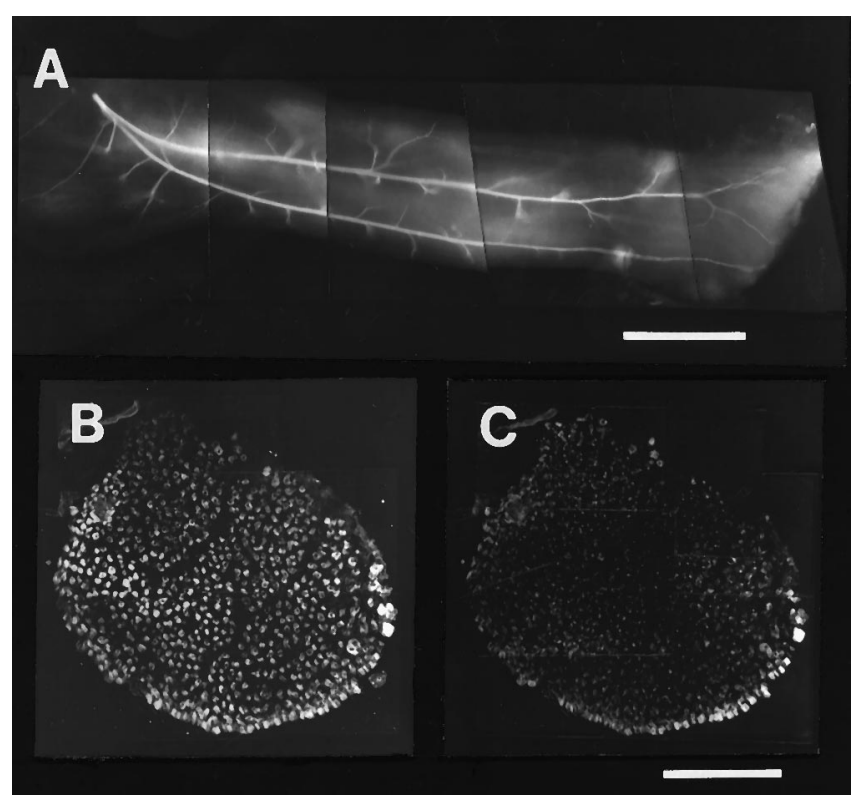

Figure 8. The distribution of intramuscular axons and relative absence of fast muscle fibers in a typical "all-slow" IFIB muscle in a rotated hindlimb. $A$, St. 36 whole-mount preparation of intramuscular nerves in an "allslow" experimental IFIB muscle shows the typical slow muscle innervation pattern with axons growing longitudinal to the muscle fibers and extending intermittent collateral sprouts. Note the complete absence of a fast, transversely oriented pattern of axon branching characteristic of the normal fast, posterior region of the muscle. Myotubes run from left (proximal) to right (distal). $B, C$, Cross section through a similar IFIB with double immunostaining for slow muscle myosin $(B)$ and fast muscle $\mathrm{Ca}^{2+}$ ATPase $(C)$ shows that the "all-slow" IFIB muscle is composed almost entirely of slow myotubes. Scale bars: $A, 500 \mu \mathrm{m} ; B, C, 200 \mu \mathrm{m}$.

Another possibility, which we consider less likely, is that axons entered muscles nonselectively, but only those that were appropriately matched to the correct muscle fiber type made functional synapses and contributed to the EMGs. To test for this possibility, after recording a typical "SART-like" EMG pattern from an "all-slow" IFIB, we cut the nerve at its entry point into the muscle and recorded, from the proximal stump, the activation pattern of all of the axons contributing to that nerve with a tight-fitting suction electrode. In control animals, neurograms recorded from the muscle nerve closely mirror the EMGs recorded from the same muscle (O'Donovan, 1989). As shown in Figure 9B, the motoneuron activation pattern recorded from the nerve was very similar to the EMGs recorded from both control SART and thin IFIB muscles (Fig. 9A). Specifically, the onset of the bursting cycle (Fig. 9B) was followed by a long period of inactivity that was similar in duration to the inhibitory period of the SART EMG. If the thin IFIB had been innervated by extensor motoneurons, the inhibitory period would have been "filled-in" with extensor-like activity even if the axons did not make functional synapses with muscle fibers. Together, these results indicate that, in the absence of a posterior fast muscle region, the IFIB in rotated limbs are exclusively innervated by flexor motoneurons belonging to the SART motoneuron pool.

\section{DISCUSSION}

\section{Selective innervation of fast and slow muscle regions}

The present study provides strong support for the existence of classes of motoneurons that early in development selectively recognize, project into, and synapse with regions of muscles contain- ing the appropriate fiber type. The experimental pITIB and "allslow" IFIB muscles were innervated by pools of motoneurons that were appropriate for the single fiber type contained in these muscles. When pools of "fast" and "slow" motoneurons innervated the same muscle (i.e., the normally formed IFIBs), they were selectively distributed to the fast and slow muscle regions. Such partitioning of foreign innervation within the IFIB has been described previously (Landmesser and O'Donovan, 1984b; Vogel and Landmesser, 1987; Vogel, 1988), but the design of those studies did not allow the authors to correlate this with fast-slow matching.

Because selective innervation of fast and slow muscle regions was found early in the motoneuron cell death period, death of inappropriately matched motoneurons cannot account for the selectivity of innervation observed. To exclude the possibility that significant numbers of motoneurons projected into regions of muscles containing inappropriate fibers, but did not form functional synapses, we recorded evoked nerve activity from an "allslow" IFIB. In this case, both the EMG and the neurogram recordings showed a clean SART-like activation pattern with no contamination from other motoneuron pools. Taken together with previous findings, that the activation pattern of motoneurons is not altered after innervating a foreign muscle (Landmesser and O'Donovan, 1984b; Vogel and Landmesser, 1987; Vogel, 1988), these results strongly suggest that developing chick muscle fibers and motoneurons have characteristic markers that facilitate the ingrowth and formation of synapses by the appropriate class of axon.

Our observations thus favor an early segregation of inputs rather than a gradual activity-based segregation as occurs during the formation of ocular dominance columns (Meyer, 1982; Boss and Schmidt, 1984). The fact that both flexor and extensor axons can be caused to project to the same muscle regions under certain experimental conditions (Vogel, 1988), and that complete blockade of motoneuron activity during the innervation process does not affect the specificity of projections (Landmesser and Szente, 1986), argues against an activity-based segregation of inputs.

\section{Motoneuron-muscle fiber matching}

Primary muscle fibers exhibit normal phenotypic differentiation in the absence of nerves (Butler et al., 1982; Phillips and Bennett, 1984; Crow and Stockdale, 1986; Condon et al., 1990b; Fredette and Landmesser, 1991) and neural activity (Fredette and Landmesser, 1991), and their distribution is spatially organized. These results suggest that, developmentally, muscle fiber phenotypes may not be determined by the particular firing pattern of the innervating motoneuron as previously proposed (e.g., Vrbova et al., 1985). Rather, they suggest that different classes of motoneurons exist and that they selectively project to and synapse with the appropriate class of already differentiated myotube (Vogel and Landmesser, 1987). However, several studies have shown that developing chick motoneurons can innervate muscle fibers of the inappropriate type (Landmesser and O'Donovan, 1984b; Vogel and Landmesser, 1987; Grim et al., 1989) and convert them to match the class of the motoneuron. How can these studies be reconciled with our observations, which show that pools of motoneurons are strongly biased to selectively innervate regions of foreign muscles containing fibers of the appropriate type? A clue to this paradox comes from the study of Grim et al. (1989).

To address whether the pattern of synapses on chick fast and slow muscle fibers is determined by the innervating motoneuron or is imposed on the nerve by the developing muscle, Grim et al. 

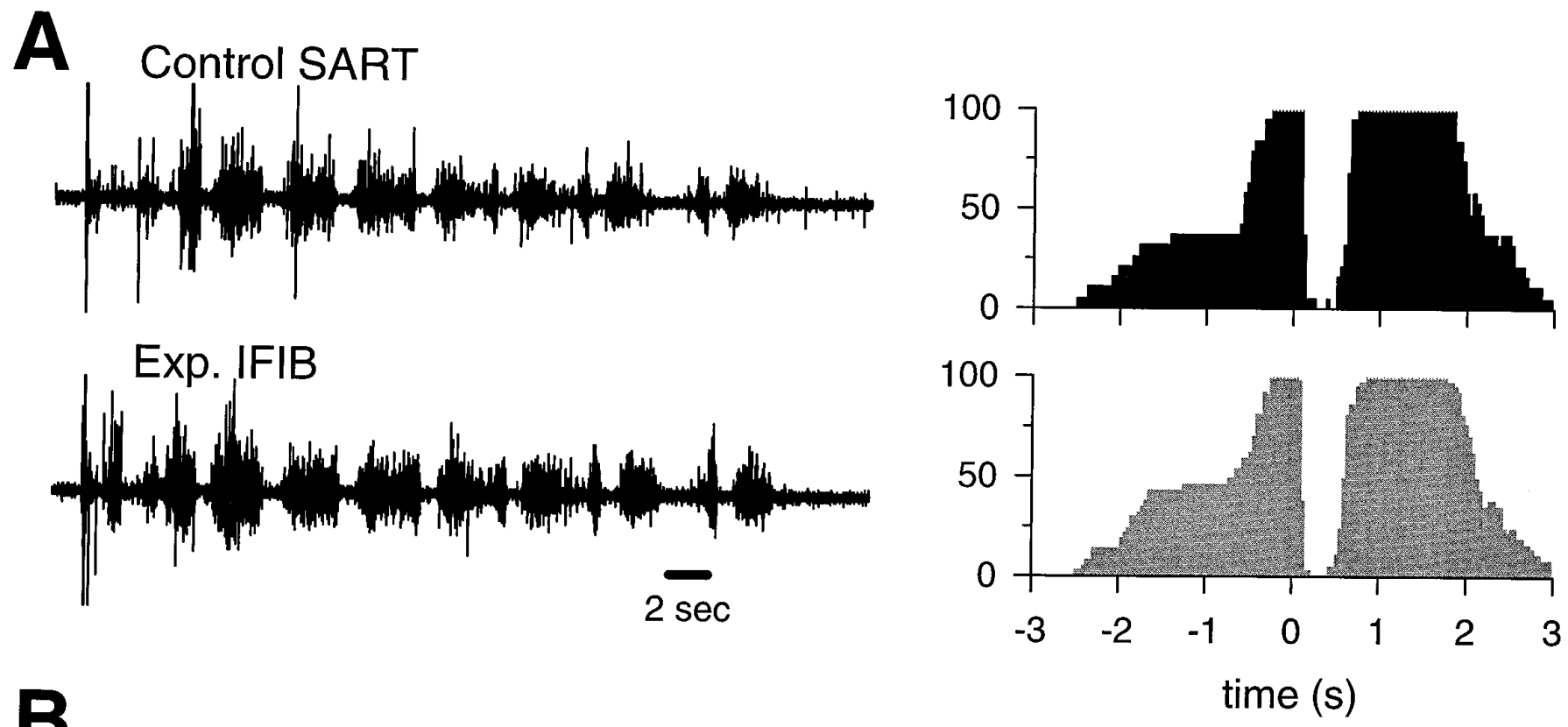

\section{Exp. IFIB Nerve}
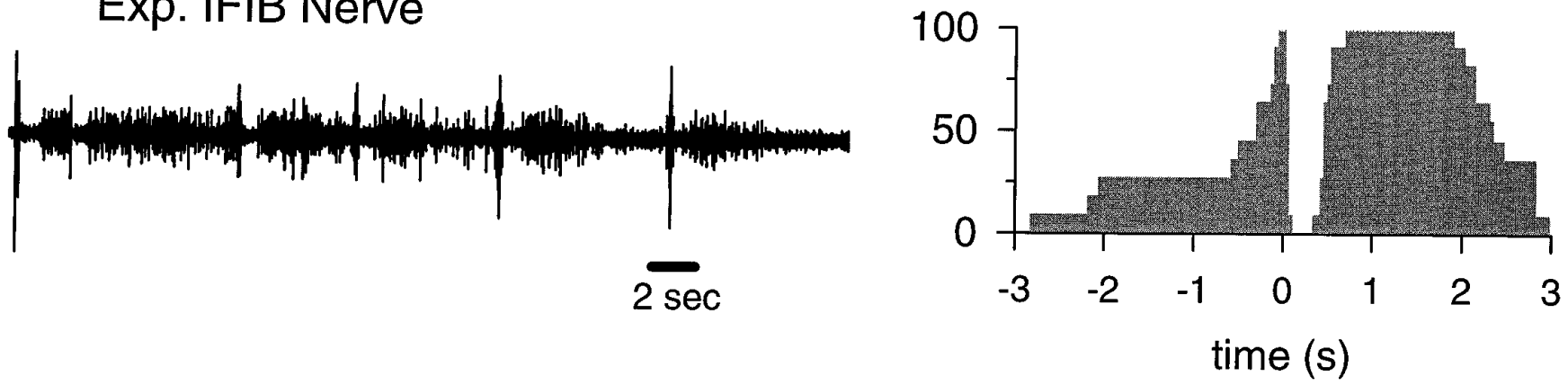

Figure 9. Typical EMG and nerve activation patterns recorded from "all-slow" IFIB muscles in rotated hindlimbs. $A$, The EMG activation pattern recorded from an IFIB lacking a fast region and the corresponding probability of activation histogram (shaded histogram) are strikingly similar to the contralateral control SART muscle (filled histogram). B, Experimental IFIB neurogram, recorded with a tight-fitting suction electrode from the proximal stump of the only nerve entering this muscle and corresponding probability of activation histogram (shaded histogram) are very similar to the EMG activation patterns recorded from both the experimental IFIB and the control SART muscles (see Fig. 2 for abbreviations).

(1989) took advantage of the fact that the chick plantaris muscle is slow whereas the quail plantaris is fast. By using interspecies hindlimb bud transplantations, they found that "slow" chick plantaris motoneurons selectively innervated quail plantaris muscles even though they were composed of fast muscle fibers. The muscle fiber phenotype and the pattern of synapses were then found to be converted to slow to match the innervating motoneurons. However, when quail limb buds were positioned at the wing level and given the chance to be innervated by a variety of foreign motoneuron pools, a selectivity similar to that described in the present study seemed to occur. The muscle fibers and end plate patterns remained fast, suggesting that the fast plantaris had been selectively innervated by fast motoneurons.

Together, these results suggest that strong environmental cues exist in the limb that selectively guide motoneurons to their correct muscles irrespective of its fiber type composition. After reaching the muscle, the axons use more local cues to selectively innervate the appropriate muscle fiber types. When innervating foreign muscles containing a mixture of fiber types, axons use these local cues to preferentially innervate the correct fibers. However, in the absence of appropriate fiber types, axons can innervate inappropriate ones and convert them to match the fast-slow nature of the motoneuron. We suggest that, in the study of Grim et al. (1989), strong muscle specific cues caused "slow" chick plantaris motoneurons to project into the all-fast quail plantaris. In addition, the fact that the distribution of muscle fiber types is strikingly similar to normal in embryonic muscles when innervated by foreign motoneurons (Laing and Lamb, 1983; Butler et al., 1986, 1988; Vogel and Landmesser, 1987; Grim et al., 1989) supports the hypothesis of selective innervation.

\section{Determinants of intramuscular nerve branching}

The intramuscular nerve branching patterns in the fast and slow regions of experimental IFIB muscles were similar to normal. The pattern of branching in these two regions is strikingly different (Dahm and Landmesser, 1988), reflecting the fact that avian slow fibers are multiply innervated whereas fast fibers are focally innervated (Ginsborg and Mackay, 1961). The factors regulating these different branching patterns are not known. Specific characteristics of fast and slow muscle fibers could impose the appropriate branching pattern on any axon that happened to innervate them. Alternatively, specific guidance cues could selectively cause "fast" and "slow" motor axons to preferentially grow into the appropriate region. Subsequent local axon-axon and/or axon- 
myotube interactions could then regulate the degree and pattern of nerve branching. Adhesive interactions between ingrowing nerves and myotubes have been shown to contribute to the extent and pattern of axon branching (Landmesser et al., 1988, 1990). Axons projecting to the fast region of the IFIB have more highly polysialylated NCAM than those innervating the slow region. Additionally, experimental removal of polysialic acid causes the branching pattern of the fast IFIB to resemble more closely the slow branching pattern (Landmesser et al., 1988, 1990). Whether foreign axons projecting to the slow and fast regions of the IFIB in the rotated limbs express different levels of polysialic acid and/or whether this can account for the observed pattern of nerve branching is currently under investigation.

\section{Mechanisms for selectivity}

Previous results support the idea that different classes of motoneurons selectively innervate muscle fibers of the appropriate type during development (Thompson et al., 1984; Gordon and Van Essen, 1985; Fladby and Jansen, 1988; Vogel and Landmesser, 1987) and regeneration (Thompson et al., 1987). At present, the cellular mechanisms involved in this matching process are unknown. Growing axons are believed to use a variety of contact-mediated and diffusible signals that are either attractive or repulsive (for review, see Landmesser, 1992; Keynes and Cook, 1995; Goodman, 1996). Several different mechanisms have been implicated to explain the matching that occurs between a motoneuron pool and its target muscle. These include selective fasciculation of like axons (Lance-Jones and Landmesser, 1981a), attraction at a distance (Lance-Jones and Landmesser, 1981b; Tosney, 1987), and active avoidance (Tosney and Oakley, 1990). It is possible that fast-slow matching also involves a variety of cellular mechanisms, including both short- and long-range and attractive as well as repulsive signals.

Axons within a motoneuron pool that project to fast and slow muscle regions selectively fasciculate with like axons in the plexus region shortly after they emerge from the spinal cord (L. Milner, V. Rafuse, and L. Landmesser, unpublished observations). This suggests that distinctive molecular differences exist at early stages of outgrowth between axons that later innervate fast and slow muscles. These could include adhesion molecules that mediate selective fasciculation as well as receptors for limb- and musclederived guidance cues. Many of our observations could be explained by axons actively avoiding the inappropriate type of muscle region, and the inhibitory molecules collapsin 1 and 2 are both present at the appropriate time (Luo et al., 1995, 1996) [see Puschel et al. (1995) for the mouse]. Although they do not seem to be differentially expressed in fast versus slow muscle, axonal receptors for them could be differentially expressed.

Semaphorins and related molecules have been shown to play a role in motor axon guidance in Drosophila (Matthes et al., 1995). In addition, eph kinases and several putative ligands have been described recently in developing motoneurons and/or mesoderm and muscle (Cheng and Flannigan, 1994; Kilpatrick et al., 1996). By analogy with the retino-tectal system (Drescher et al., 1995; Cheng et al., 1995), it has been suggested that they may be mediating repulsive guidance interactions. Finally, although we have no data that directly support attractive signals in fast-slow matching, these could be present, acting as either secreted and/or membrane-bound signals. It will be interesting to explore the molecular basis of fast-slow matching and to confirm that fast and slow motoneurons have distinct molecular differences as inferred from the results in the present study.

\section{REFERENCES}

Acheson A, Sunshine JL, Rutishauser U (1991) NCAM polysialic acid can regulate both cell-cell and cell-substrate interactions. J Cell Biol 114:143-154.

Boss V, Schmidt J (1984) Activity and the formation of ocular dominance patches in dually innervated tectum of goldfish. J Neurosci 4:2891-2905.

Brown MC, Booth CM (1983) Postnatal development of the adult pattern of motor axon distribution in rat muscle. Nature 304:741-742.

Butler J, Cauwenbergs P, Cosmos E (1986) Fate of brachial muscles of the chick embryo innervated by inappropriate nerves: structural, functional and histochemical analysis. J Embryol Exp Morphol 95:147-168.

Butler J, Cosmos E, Cauwenbergs P (1988) Positional signals: evidence for a possible role in muscle fibre-type patterning of the embryonic avian limb. Development 102:763-772.

Cheng H-J, Flanagan JG (1994) Identification and cloning of elf-1, a developmentally expressed ligand for the Mek4 and Sek receptor tyrosine kinases. Cell 79:157-168.

Cheng H-J, Nakamoto M, Bergemann AD, Flanagan JG (1995) Complementary gradients in expression and binding of the topographic retinotectal projection map. Cell 82:371-381.

Condon K, Silberstein L, Blau HM, Thompson WJ (1990a) Development of muscle fiber types in the prenatal rat hindlimb. Dev Biol 138:256-274.

Condon K, Silberstein L, Blau HM, Thompson WJ (1990b) Differentiation of fiber types in aneural musculature of the prenatal rat hindlimb. Dev Biol 138:275-295.

Crow MT, Stockdale FE (1986) Myosin expression and specialization among the earliest muscle fibers of the developing avian limb. Dev Biol 113:238-254.

Dahm LS, Landmesser LT (1988) The regulation of intramuscular nerve branching during normal development and following activity blockade. Dev Biol 130:621-644.

Drescher U, Kremoser C, Handwerker C, Loschinger J, Noda M, Bonhoeffer $F$ (1995) In vitro guidance of retinal ganglion cell axons by RAGS, a $25 \mathrm{kDa}$ tectal protein related to ligands for Eph receptor tyrosine kinases. Cell 82:359-370.

Fladby T, Jansen JKS (1988) Selective innervation of neonatal fast and slow muscle fibres before net loss of synaptic terminals in the mouse soleus muscle. Acta Physiol Scand 134:561-562.

Fredette BJ, Landmesser LT (1991) A reevaluation of the role of innervation in primary and secondary myogenesis in developing chick muscles. Dev Biol 143:19-35.

Frelinger AL, Rutishauser U (1986) Topology of NCAM structural and functional determinants. II. Placement of monoclonal antibody epitopes. J Cell Biol 103:1729-1737.

Ginsborg BL, Mackay B (1961) A histochemical demonstration of two types of motor innervation in avian skeletal muscle. Bibl Anat 2:174-181.

Goodman CS (1996) Mechanisms and molecules that control growth cone guidance. Annu Rev Neurosci 19:342-377.

Gordon H, Van Essen DC (1985) Specific innervation of muscle fiber types in a developmentally polyneuronally innervated muscle. Dev Biol 11:42-50.

Grim M, Nensa K, Christ B, Jacob HJ, Tosney KW (1989) A hierarchy of determining factors controls motoneuron innervation: experimental studies on the development of the plantaris muscle (PL). Anat Embryol 180:179-189.

Grinnell AD (1995) Dynamics of nerve-muscle interaction in developing and mature neuromuscular junctions. Physiol Rev 75:789-834.

Hamburger V, Hamilton HL (1951) A series of normal stages in the development of the chick embryo. J Morphol 88:49-92.

Kaprielian Z, Fambrough DM (1987) Expression of fast and slow isoforms of the $\mathrm{Ca}^{2+}$-ATPase in developing chick skeletal muscle. Dev Biol 124:490-503.

Kernell D (1992) Organized variability in the neuromuscular system. Arch Ital Biol 130:19-66.

Keynes R, Cook GMW (1995) Axon guidance molecules. Cell 83:161-169.

Kilpatrick TJ, Brown A, Lai C, Gassmann M, Goulging M, Lemke G (1996) Expression of the Tyro4/Mek4/Cek4 gene specifically marks a subset of embryonic motor neurons and their muscle targets. Mol Cell Neurosci 7:62-74.

Laing NG, Lamb AH (1983) The distribution of muscle fibre types in chick embryo wings transplanted to the pelvic region is normal. J Embrol Exp Morphol 78:67-82. 
Lance-Jones C, Landmesser L (1980) Motoneurone projection patterns in the chick hind limb following early partial reversals of the spinal cord. J Physiol (Lond) 302:581-602.

Lance-Jones C, Landmesser L (1981a) Pathway selection by chick lumbosacral motoneurons during normal development. Proc R Soc Lond [Biol] 214:1-18.

Lance-Jones C, Landmesser L (1981b) Pathway selection by embryonic chick motoneurons in an experimentally altered environment. Proc $\mathrm{R}$ Soc Lond [Biol] 214:19-52.

Landmesser L (1978) The distribution of motoneurones supplying chick hind limb muscles. J Physiol (Lond) 284:371-389.

Landmesser LT (1992) Growth cone guidance in the avian limb: a search for cellular and molecular mechanisms. In: The nerve growth cone (Letourneau PC, Kater SB, Macagno ER, eds), pp 373-385. New York: Raven.

Landmesser L, O'Donovan M (1984a) Activation patterns of embryonic chick hindlimb muscles recorded in ovo and in an isolated spinal cord preparation. J Physiol (Lond) 347:189-204.

Landmesser L, O'Donovan M (1984b) The activation patterns of embryonic chick motoneurons projecting to inappropriate muscles. J Physiol (Lond) 347:205-224.

Landmesser LT, Szente M (1986) Activation patterns of embryonic chick hind-limb muscles following blockade of activity and motoneurone cell death. J Physiol (Lond) 380:157-174.

Landmesser L, Dahm L, Schultz K, Rutishauser U (1988) Distinct roles for adhesion molecules during innervation of embryonic chick muscle. Dev Biol 130:645-670.

Landmesser L, Dahm L, Tang J, Rutishauser U (1990) Polysialic acid as a regulator of intramuscular nerve branching during embryonic development. Neuron 4:655-667.

Laskowski MB, Sanes JR (1987) Topographical mapping of motor pools onto skeletal muscles. J Neurosci 7:252-260.

Luo Y, Shepherd I, Li J, Renzi MJ, Chang S, Raper JA (1995) A family of molecules related to collapsin in the embryonic chick nervous system. Neuron 14:1131-1140.

Matthes DJ, Sink H, Kolodkin AK, Goodman CS (1995) Semaphorin II can function as a selective inhibitor of specific synaptic arborizations. Cell 81:631-639.

McLennan I (1983) Differentiation of muscle fiber types in the chicken hindlimb. Dev Biol 97:222-228.

Meyer R (1982) Tetrodotoxin blocks the formation of ocular dominance columns in goldfish. Science 218:589-591.
O'Donovan MJ (1989) Motor activity in the isolated spinal cord of the chick embryo: Synaptic drive and firing pattern of single motoneurons. J Neurosci 9:943-958.

Phillips WD, Bennett MR (1984) Differentiation of fiber types in wing muscles during development: effect of neural tube removal. Dev Biol 106:457-468.

Puschel AW, Adams RH, Betz H (1995) Murine semaphorin C/collapsin is a member of a diverse gene family and creates domains inhibitory for axonal extension. Neuron 14:941-948.

Rafuse VF, Milner L, Landmesser L (1995) Determinants of intramuscular axon branching in developing chick muscle. Soc Neurosci Abstr 12:124.3.

Shepherd I, Luo Y, Raper JA, Chang S, (1996) The distribution of collapsin-1 mRNA in the developing chick nervous system. Dev Biol 173:185-199.

Swett JE, Elred E, Buchwald JS (1970) Somatotopic cord-to-muscle relations in efferent innervation of cat gastrocnemius. Am J Physiol 219:762-766.

Thompson WJ, Sutton LA, Riley DA (1984) Fibre type composition of single motor units during synapse elimination of neonatal rat soleus muscle. Nature 309:709-711.

Thompson WJ, Soileau LC, Balice-Gordon RJ, Sutton LA (1987) Selective innervation of types of fibers in developing rat muscles. $\mathrm{J}$ Exp Biol 132:249-263.

Tosney KW (1987) Proximal deleions and patterned neurite outgrowth at the lumbosacral level of the chick embryo: deletion of the dermamyotome. Dev Biol 122:540-588.

Tosney K, Oakley R (1990) The perinotochordal mesenchyme acts as a barrier to axon advance in the chick embryo: implications for a general mechanism of axon guidance. Exp Neurol 109:75-79.

Vogel M (1988) Activation patterns of embryonic chick lumbosacral motoneurons following large spinal reversals. J Physiol (Lond) 389:491-512.

Vogel M, Landmesser L (1987) Distribution of fiber types in embryonic chick limb muscles innervated by foreign motoneurons. Dev Biol 119:481-495.

Vrbova G, Navarrete R, Lowrie M (1985) Matching of muscle properties and motoneurone firing patterns during early stages of development. J Exp Biol 115:113-123.

Vrbova G, Gordon T, Jones R (1995) Nerve-muscle interaction. London: Chapman \& Hall. 